\title{
Effects of Residual Moisture and Zero Conditioning Time on Maximum Theoretical Specific Gravity
}

John Elias Crane

West Virginia University

Follow this and additional works at: https://researchrepository.wvu.edu/etd

\section{Recommended Citation}

Crane, John Elias, "Effects of Residual Moisture and Zero Conditioning Time on Maximum Theoretical Specific Gravity" (2012). Graduate Theses, Dissertations, and Problem Reports. 3367.

https://researchrepository.wvu.edu/etd/3367

This Thesis is protected by copyright and/or related rights. It has been brought to you by the The Research Repository @ WVU with permission from the rights-holder(s). You are free to use this Thesis in any way that is permitted by the copyright and related rights legislation that applies to your use. For other uses you must obtain permission from the rights-holder(s) directly, unless additional rights are indicated by a Creative Commons license in the record and/ or on the work itself. This Thesis has been accepted for inclusion in WVU Graduate Theses, Dissertations, and Problem Reports collection by an authorized administrator of The Research Repository @ WVU. For more information, please contact researchrepository@mail.wvu.edu. 


\title{
Effects of Residual Moisture and Zero Conditioning Time on Maximum Theoretical Specific Gravity
}

\section{John Elias Crane}

Thesis submitted to the College of Engineering and Mineral Resources at West Virginia University in partial fulfillment of the requirements for the degree of

Master of Science

In

Civil Engineering

\author{
Dr. John P. Zaniewski, Chair \\ Dr. John Quaranta \\ Dr. Avinash Unnikrishnan \\ Department of Civil and Environmental Engineering \\ Morgantown, West Virginia \\ May, 2012
}

Keywords: Slag, asphalt mix design, theoretical maximum gravity, $\mathrm{G}_{\mathrm{mm}}$, Dry Back 


\section{ABSTRACT \\ Effects of Residual Moisture and Zero Conditioning Time on Maximum Theoretical Specific Gravity}

\section{John Elias Crane}

A critical issue in determining theoretical maximum specific gravity $\left(\mathrm{G}_{\mathrm{mm}}\right)$ for Hot Mix Asphalt (HMA) mixtures appears when the aggregates used have high absorption. This high absorption in aggregates, like air cooled iron blast furnace slag, can greatly affect the $G_{m m}$ values of the sample, which in turn affects the contractor's ability to accurately evaluate the volumetric properties of the asphalt concrete. WV Paving, the largest asphalt paving contractor in West Virginia, uses slag aggregates in many of their mixes. There is concern that the dry-back procedure used to determine $G_{m m}$ of these mixtures is not producing reliable results. $G_{m m}$ results from lab prepared mixes were observed to be different from plant prepared mixes. This is of concern to both the contractor and the West Virginia Division of Highways as $\mathrm{G}_{\mathrm{mm}}$ is an important parameter in both mix design and quality control. Two hypotheses for the issue were identified that may affect the measured properties of the plant produced mixes; residual moisture and a short conditioning time. To test the effects of residual moisture samples of slag aggregate were saturated with water for a period of time then heated allowing some of the water to evaporate and then mixed with other aggregates and binder. Samples were also produced and tested without having the two hour conditioning time required in the AASHTO procedures. Findings indicated that a third of the residual moisture samples were significantly different from the control values, with seventy percent of those samples containing high slag contents. The conditioning time results showed that the lack of a conditioning period was significantly different than the controlled two hour conditioning at a 95\% confidence level.

A related issue is a vacuum method for measuring $G_{m m}$ has been developed and is in use by some contractors. The experimental design for this research compared the results of the standard test method to the vacuum method. There is a small, but consistent difference between the standard and vacuum methods. Further research should be conducted to see if a short conditioning time should be required for HMA mixtures containing highly absorptive aggregates. 


\section{TABLE OF CONTENTS}

Abstract $\quad$ ii

List of Figures $\quad$ V

List of Tables vi

Chapter 1 Introduction 1

1.1 Background 1

1.2 Problem Statement 1

1.3 Objective 2

1.4 Organization of Thesis 2

Chapter 2 Literature Review 4

2.1 Introduction 4

2.2 Slag in HMAC $\quad 4$

2.2.2 Different Types of Slag 5

2.2.3 Benefits of ACBFS

2.2.4 Drawbacks of ACBFS 6

2.3 Test Methods 6

$\begin{array}{ll}\text { 2.3.1 CoreLok } & 6\end{array}$

2.3.2 Rice Method and Additional Dry-Back Procedures 9

2.4 $\mathrm{G}_{\mathrm{mm}}$ investigations Using the CoreLok 11

2.4.1 Florida DOT 11

2.4.2 University of Cincinnati 13

Chapter 3 Research Methodology 16

3.1 Introduction 16

3.2 Experimental Design $\quad 16$

3.3 Material 17

$\begin{array}{ll}\text { 3.3.1 Asphalt Cement } & 17\end{array}$

$\begin{array}{ll}\text { 3.3.2 Aggregate Properties } & 18\end{array}$

$\begin{array}{ll}\text { 3.3.3 Aggregate Preparation } & 18\end{array}$

$\begin{array}{ll}3.4 \text { Mix Design } & 19\end{array}$

3.4.1 Aggregate Blends $\quad 19$

3.5 Sample Creation 20

3.5.1 Additional Procedures for Water Addition $\quad 22$

3.5.1 Additional Procedures for Zero Conditioning Time 24 
3.6 Sample Testing $\quad 24$

3.6.1 Test Methods $\quad 24$

Chapter 4 Results and Analysis $\quad 26$

4.1 Introduction $\quad 26$

4.2 Results $\quad 27$

4.2.1 $G_{m m}$ Analysis of Test Methods $\quad 28$

4.2.2 Analysis of Conditioning Time $\quad 32$

4.2.3 $G_{\text {se }}$ Analysis $\quad 36$

4.3 Comparison to Previous Research 41

Chapter 5 Conclusions and Recommendations 45

5.1 Conclusions $\quad 45$

5.2 Recommendations for Further Study 46

$\begin{array}{ll}\text { References } & 49\end{array}$

Appendix - Data Sheets $\quad 50$ 


\section{LIST OF FIGURES}

Figure 1 - The CoreLok Vacuum Machine with the Agg. Plus Device 7

Figure 2 - CoreLok Procedure Step A: 1500 g placed in the channel bag. $\quad 7$

Figure 3 - CoreLok Procedure Step B: Channel bag placed inside larger bag. 8

Figure 4 - CoreLok Procedure Step C: Both bags and sample are placed in the CoreLok, the lid is closed. After the vacuum and sealing processes are completed the bags can be removed.

Figure 5 - Data Chart for CoreLok $\mathrm{G}_{\mathrm{mm}}$ Test 10

Figure 6- Test set up for FDOT test method FM 1-T209 12

Figure 7 - Combined Mixture Gradations $\quad 21$

Figure 8 - Drying Rack $\quad 25$

Figure 9 - Line of Equality Chart for Rice versus CoreLok $G_{m m}$ Results. 30

Figure 10 - Line of Equality Chart for Dry Back versus Rice $G_{m m}$ Results. 33

Figure 11 - Adjusted Line of Equality Chart for Dry Back versus Rice $\mathrm{G}_{\mathrm{mm}}$ $\begin{array}{ll}\text { Results } & 34\end{array}$

Figure 12 - Line of Equality Chart for Dry Back versus CoreLok $G_{m m}$ Results. 34

Figure 13 - Conditioning Time Means 35

Figure $14-\mathrm{G}_{\mathrm{se}}$ Means $\quad 38$

Figure 15 - $\mathrm{G}_{\mathrm{se}}$ Test Data versus Slag Content 39

Figure 16 - Line of Equality for Dry vs. Moist sample comparison 42

Figure 17 - Moisture Addition Line of Regression for Zero conditioned samples 42

Figure 18 - Line of Equality on Rajagopal and Crago study 44 


\section{LIST OF TABLES}

Table 1- FDOT t-test Results for $\mathrm{G}_{\mathrm{mm}}$ Test Data for the CoreLok and FM 1-T 209

Table 2 - Comparison Data for AASHTO T-209 and CoreLok (Rajagopal and Crago 2007)

Table 3 - Experimental Factors/Levels

Table 4 - Data Table

Table 5 - Aggregate Properties

Table 6 - Aggregate Gradations

Table 7 - Combined Mixture Gradations \& Properties

Table 8-Example Aggregate Weigh-out Table

Table 9 - Experimental Factors and Levels

Table 10 - Description of Acronyms

Table $11-\mathrm{G}_{\mathrm{mm}}$ Test Results

Table 12 - $\mathrm{G}_{\mathrm{mm}}$ Means and Standard Deviations

Table 13 - t-test comparisons of test methods

Table 14 - Results of Regression Analysis for Rice versus CoreLok

Table 15 - $\mathrm{G}_{\mathrm{se}}$ Data Sheet

Table 16 - $\mathrm{G}_{\text {se }}$ Means and Standard Deviations

Table 17 - Slag Content Analysis Using $\mathrm{G}_{\mathrm{se}}$

Table 18 - $\mathrm{G}_{\mathrm{se}}$ Comparison for Additional Binder

Table $19-\mathrm{G}_{\mathrm{mm}}$ Moisture Addition t-test Table

Table 20 - Regression analysis on Rajagopal and Crago Study

Table 21 - Summary of Analytical Tests 
Table 23 - MD DATA

Table 24 - HD DATA

Table 25 - LDM DATA

Table 26 - MDM DATA

Table 27 - HDM DATA 55

Table 28 - LZ DATA 56

Table 29 - MZ DATA 57

Table 30 - HZ DATA 58

Table 31 - LZM DATA 59

Table 32 - MZM DATA 60

Table 33 - HZM DATA

Table 34 - LP DATA

Table 35 - MP DATA 63

Table 36 - HP DATA 64

Table 37 - LPM DATA 65

Table 38 - MPM DATA 66

Table 39 - HPM DATA 


\section{CHAPTER 1 INTRODUCTION}

\subsection{BACKGROUND}

In order to correctly evaluate asphalt concrete’s volumetric properties the theoretical maximum specific gravity $\left(\mathrm{G}_{\mathrm{mm}}\right)$ must be accurately measured. This has proven to be a problem when absorptive aggregates are used in a mixture. The dry-back procedure has been implemented for years in attempt to correct this issue; however this procedure is prone to errors. In more recent years a new device, the CoreLok, has been released as an alternative method for measuring specific gravity of asphalt mixes.

One of the significant problems with the use of certain aggregates is their absorptive properties. Once a sample of asphalt is mixed, the longer it conditions the more asphalt is absorbed into the aggregates until the voids are saturated with asphalt. This absorption greatly changes the $G_{m m}$ value of the sample. This is then reflected in the values for air voids and makes it more difficult for a contractor to achieve their correct densities in the field while constructing a new pavement.

The need for improving specific gravity measurements is not unique to West Virginia. The FHWA (2010) reviewed methods for determining specific gravity and the associated impact on volumetric analysis of asphalt mixes. With respect to $G_{m m}$ the FHWA report states:

The current standard test methods for determination of Gmm for HMA mixtures containing aggregate with low absorption are satisfactory. However, the multilaboratory precision estimate for mixtures containing moderately to highly absorptive aggregate is so large that it is not valid to distinguish air voids results for split specimens conducted in two laboratories that differ by as much as 2.0 percent. Clearly, further work needs to be conducted to improve the reproducibility of the $\mathrm{Gmm}$ determination for such aggregate. Another important objective for further research should be to reduce the time to complete the test for mixes containing absorptive aggregate.

\subsection{PROBLEM STATEMENT}

The material issue evaluated in the research is from an asphalt plant in the southern part of West Virginia. West Virginia Paving, Dunbar, WV, has been having 
issues with theoretical maximum specific gravity test results between laboratory produced samples and samples taken from plant production. Plant produced samples have lower $G_{m m}$ values compared to those created in the laboratory for mix design. Two main questions arose from discussions about this problem. Is there a small amount of water still inside the absorptive material after it exits the asphalt plant? In this case the water may evaporate out of the stone leaving small micro punctures in the asphalt coating. Second, plant samples are pulled straight from the plant and tested; is there sufficient time between mixing and testing to allow the asphalt to absorb into the voids of the slag?

In addition, a vacuum based procedure for measuring asphalt mix specific gravity has been developed and is being used by some contractors. This method uses the CoreLok system developed by Instrotek, Inc. The WVDOH has limited experience with the CoreLok method. To gain experience with this test method, samples were evaluated with the conventional (Rice) method, the CoreLok method and the dry-back process.

\subsection{OBJECTIVE}

The objectives for this research were all observed to aid in determining a quality answer for the theoretical maximum specific gravity. The three objectives are as followed:

- Is the Dry Back procedure necessary?

- $\quad$ Can the CoreLok replace the original Rice Test (AASHTO’s T209)?

- Is there a difference between the plant and laboratory processes?

Null hypotheses were used to distinguish if there was any difference between the test methods and between the different sample variations described above.

\subsection{ORGANIZATION OF THESIS}

This thesis is organized into five chapters. Following the introduction, Chapter 2 contains background on the use of slag in hot mix asphalt pavements, brief history on the T209 and CoreLok test methods, and finally summaries of research conducted with the CoreLok. Chapter 3 discusses the research methodologies and test procedures that were used in the laboratory to conduct the research. Chapter 4 contains the analysis of the 
results from the laboratory findings. Finally in Chapter 5 conclusions and recommendations are presented. The Appendix includes the test results for each mixture. 


\section{CHAPTER 2 LITERATURE REVIEW}

\subsection{INTRODUCTION}

With the great quantities of waste products created each year there is a strong push to find better uses for them. The transportation sector has been a great recipient of these materials. Currently the Federal Highway Administration (FHWA) allows many different kinds of by-products to be used in the construction of asphalt pavements; the list below illustrates the wide span of materials.

Blast Furnace Slag

Coal Bottom Ash

Coal Boiler Slag

Foundry Sand

Mineral Processing Wastes

Municipal Solid Waste Combustor Ash

\author{
Nonferrous Slags \\ Reclaimed Asphalt Pavement \\ Roofing Shingle Scrap \\ Scrap Tires \\ Steel Slag \\ Waste Glass
}

While there are many allowable types of materials this research evaluated the use of Blast Furnace Slag, therefore the remaining materials will not be mentioned further in this report.

\subsection{SLAG IN HMAC}

Blast Furnace slag has become an important resource in many states for the use in road construction and rehabilitation. At least 17 states have adopted the use of blast furnace slag; several are located in the eastern United States. According to the US Geological Survey in 2006 nearly 41.3\% of air-cooled blast furnace slag was used for road bases and surfaces, another $13.3 \%$ was used for asphaltic concrete (Van Oss, 2007).

Slag is a co-product of the production of iron and steel, along with other metals. For the production of iron and steel, iron ore, scrap iron and steel, fluxes (limestone and/or dolomite), and coke are placed in the blast furnace. The coke is then combusted to reduce the iron ore to a molten state. The slag which primarily consists of silicates, alumina-silicates, and calcium-alumina-silicates is less dense then iron therefore it floats on the molten iron and can be separated from the iron product and removed as a coproduct. Although slag is less dense than iron slag's density greatly depends on the 
residual iron content in the slag. Typical specific gravities range from 2.0 to 2.5 for air cooled slag (FHWA, 1997).

\subsubsection{Different Types of Slag}

Once the slag is formed in the blast furnace there are multiple ways to separate the slag, which creates different products. The main methods are Air-Cooling, Expanded or Formed, and Pelletized.

Air-cooled blast furnace slag (ACBFS) forms from the cooling of liquid slag that is poured into large beds and is allowed to cool slowly under normal conditions. This slow cooling allows the slag to form a hard crystalline structure. After the slag cools it is crushed into usable sized aggregates.

Expanded slag is cooled with the use of water, steam, or air. This process allows for an accelerated cooling which increases the size of the crystalline structure, leaving a lightweight product. Expanded slag has a much higher porosity and lower specific gravities if compared to air cooled slag.

Pelletized slag is formed when molten slag is quenched with water or air in a spinning drum. The process forms pellets which can be controlled by the speed of the quenching process. If the slag is rapidly cooled, less crystallization will occur and the slag will have a glassy appearance (FHWA, 1997).

Air-cooled blast furnace slag will be discussed from this point on since it was used for this experiment.

\subsubsection{Benefits of ACBFS}

The main benefits from the use of slag are some of its physical and mechanical properties. ACBFS is angular with a roughly cubical shape. ACBFS also has good frictional resistance, stripping resistance, and high stability. Slags high stability is due to its high internal angle of friction, which is 40 to 45 degrees. The rough, porous, angular surface and hardness (5 to 6) of slag all help contribute to a high frictional resistance. The slag used in West Virginia has been approved as a skid resistant aggregate suitable for use in wearing layers. Slag also has high polished stone values and an affinity to asphalt, not water, and therefore, slag has high stripping resistance (FHWA, 1997). 


\subsubsection{Drawbacks of ACBFS}

The two main flaws with the use of slag is its high absorption from its porous surface, and the high variability in the material. The FHWA states that since there is variation in the production process of iron that variability continues into the slag, resulting in inconsistent results for gradations, specific gravities, absorptions, and angularity. This lack of consistency contributes to HMAC performance problems, like flushing, raveling, and high fines. Flushing is related to high binder content and raveling is related to low binder content which could be from the inconsistency in the absorption of ACBFS. Absorption is the other main drawback with slag. A high absorption requires more asphalt to be added to the mixture, which adds cost to the mixture. The FHWA says that this cost is usually offset by the high yield from using a slag mixture. The high yield is due to the lower density of the slag, therefore more volume for the same amount of weight (FHWA, 1997).

\subsection{TEST METHODS}

\subsubsection{CoreLok}

The CoreLok was developed by InstroTek Inc. located in Raleigh, North Carolina and was released in the late 1990's. InstroTek developed this vacuum sealing device to address the limitations of the previous test methods. The CoreLok is not only used for testing specific gravities for asphalt samples, it can test a wide variety of samples. This includes apparent specific gravity, absorption, and bulk specific gravity of aggregates using the AggPlus system. The CoreLok can also test for porosity of compacted asphalt samples and can be used to determine the percentage of asphalt in a mixture. Figure 1 illustrates the CoreLok and the additional AggPlus System (InstroTekCorelok Aggregate, 2011).

The basic procedure for using the machine is: the sample is weighed and placed in a sample or channel bag, for a $\mathrm{G}_{\mathrm{mm}}$ sample ASTM requires at least 1500 grams be used. The sample size does not change with the nominal maximum aggregate size like AASHTO's T209. The sample and bag are then placed inside of a larger plastic bag and everything is placed into the CoreLok chamber, shown in Figure 2, 3, and 4. The vacuum is then applied, which is approximately $30 \mathrm{in.} \mathrm{Hg}$ (InstroTekCorelok Operations, 2011). 


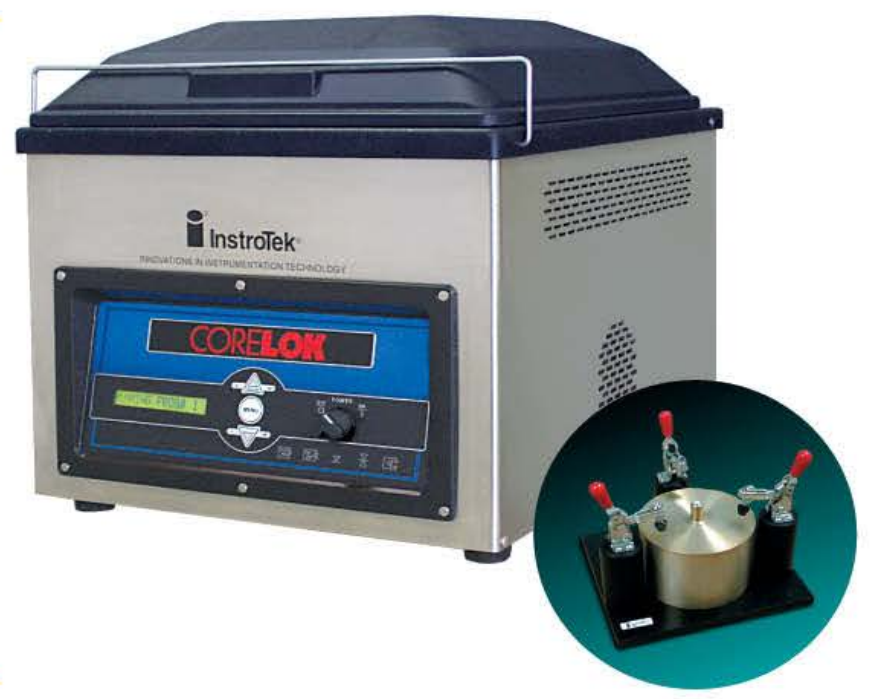

Figure 1 - The CoreLok Vacuum Machine with the Agg. Plus Device

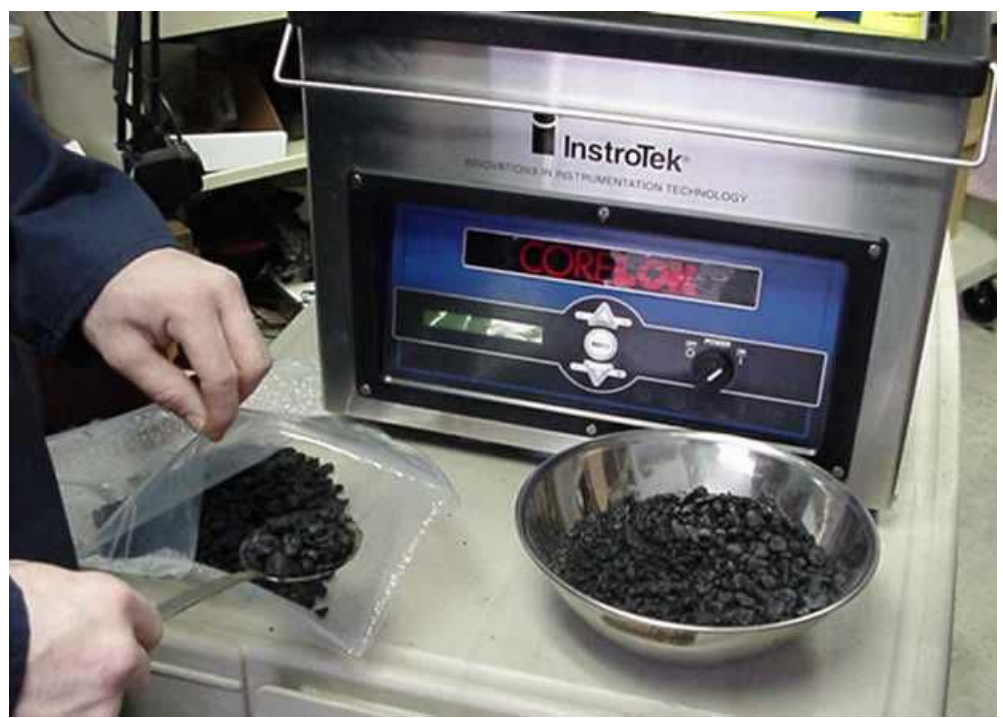

Figure 2 - CoreLok Procedure Step A: 1500 g placed in the channel bag. 


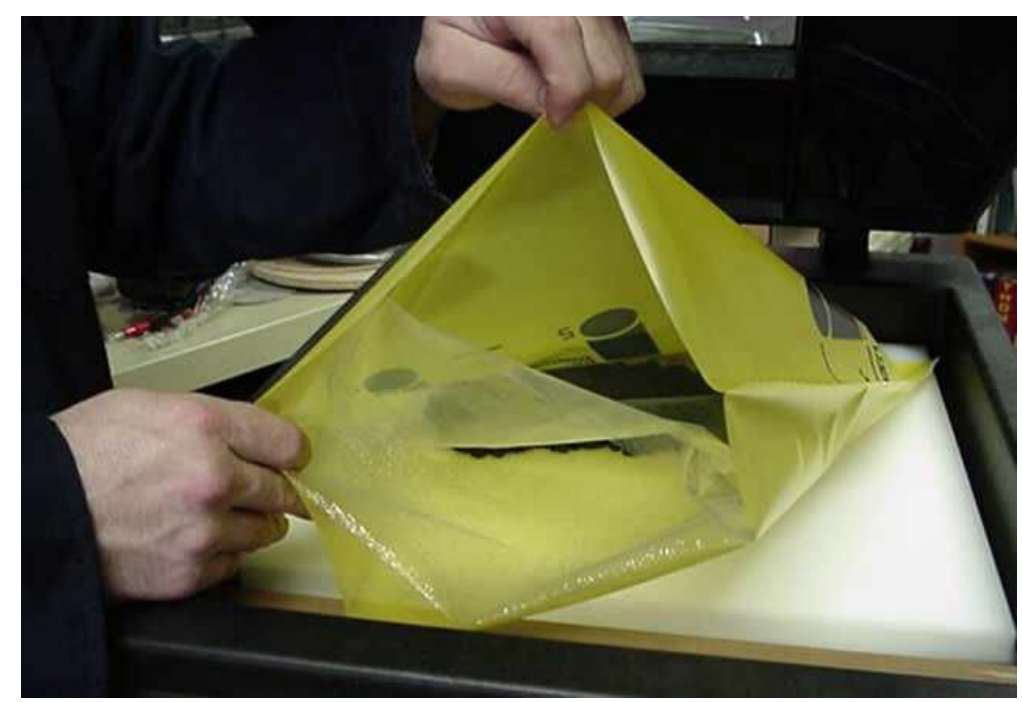

Figure 3 - CoreLok Procedure Step B: Channel bag placed inside larger bag.

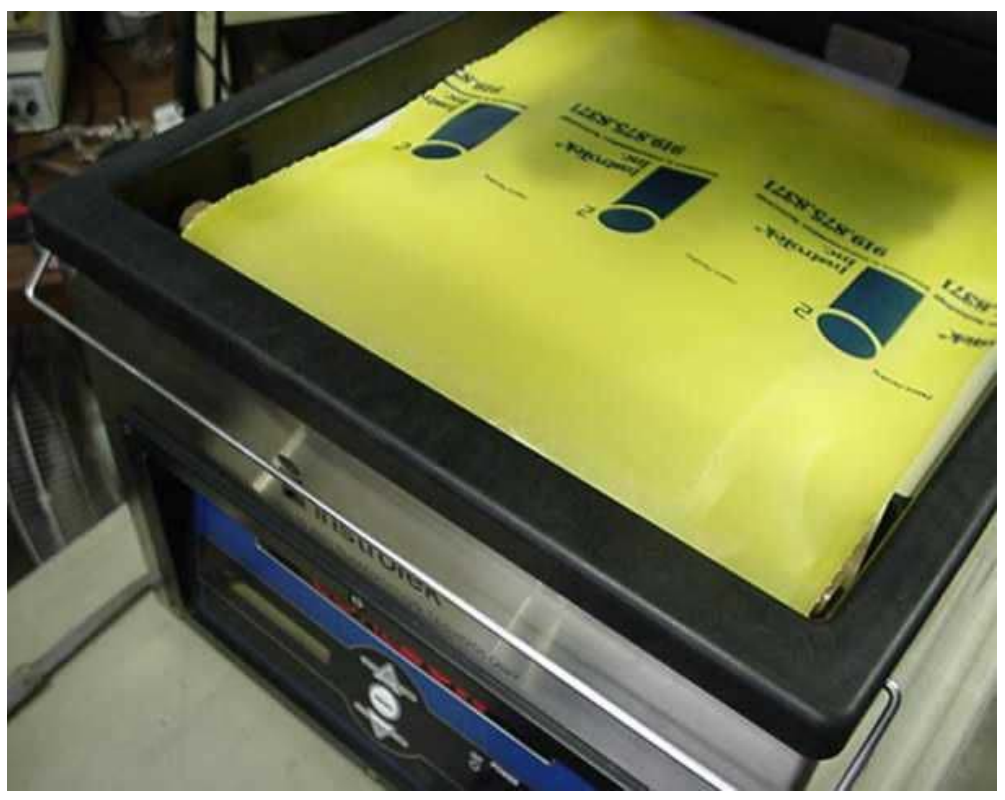

Figure 4 - CoreLok Procedure Step C: Both bags and sample are placed in the CoreLok, the lid is closed. After the vacuum and sealing processes are completed the bags can be removed.

After the vacuum is drawn it is maintained for a predetermined dwelling time depending on the type of sample tested. For a $\mathrm{G}_{\mathrm{mm}}$ sample the dwelling time is five minutes. Following the dwelling time, the outer bag is sealed and the vacuum is released. The bag is removed from the chamber and transferred to a water bath, then depending on the test being performed the bag is cut open and water is allowed to saturate the sample. 
Weights are recorded and used to calculate the desired value. InstroTek provides data sheets for individual tests along with a computer program that will return the desired value; Figure 5 is the data sheet for the $\mathrm{G}_{\mathrm{mm}}$ procedure. There are other equipment and extra procedures if doing tests with the AggPlus system.

For the experiment presented herein, only $\mathrm{G}_{\mathrm{mm}}$ samples were tested. For a $\mathrm{G}_{\mathrm{mm}}$ sample the weights needed for the calculation are the dry weight, the weight of the bags (channel and large), and the weight submerged under water. The formula for calculating the $\mathrm{G}_{\mathrm{mm}}$ value is (InstroTek, 2011):

$$
G_{m m}=\frac{B}{(A+B-C)-\left(A / V_{C}\right)}
$$

Where:

A=Bag weight

$\mathrm{B}=$ Weight of Sample in Air

$\mathrm{C}=$ Submerged Weight of Sample in Water

$\mathrm{V}_{\mathrm{C}}=$ Density of Bags $=0.903 \mathrm{~g} / \mathrm{cm}^{3}$

InstroTek claims that when testing maximum specific gravity there is no need for the dry-back method because of the limited amount of time the sample is exposed to water. Also InstroTek designed the CoreLok so there was no need to vibrate the sample as it was drawing a vacuum; they say this vibrating will potentially strip the asphalt from the rocks.

\subsubsection{Rice Method and Additional Dry-Back Procedures}

The Rice method, AASHTO T 209, was developed by James Rice in 1964. This procedure is used to determine the theoretical maximum specific gravity of an uncompact asphalt sample, which resembles the specific gravity of the mix if compacted so it contained no air voids. In the early 1990's an improved Rice method was developed and an additional dry-back procedure was added for highly absorbent aggregates. The full description of the procedure is found in the AASHTO's Standard Specifications for Transportation Materials, Part 2A: Tests, T-209. 


\section{CoreLok ${ }^{\mathrm{TM}}$ Max/Apparent Gravity Data Collection Table}

\begin{tabular}{|c|c|c|c|c|c|c|c|c|}
\hline Sample \# & $\begin{array}{c}\text { A } \\
\text { Bag Weight } \\
\text { (grams) }\end{array}$ & $\begin{array}{c}\text { B } \\
\text { Weight of } \\
\text { Rubber Sheets } \\
\text { (grams) } \\
\text { (put in } 0 \text { if not } \\
\text { used) }\end{array}$ & $\begin{array}{c}\mathrm{C} \\
\text { Weight of } \\
\text { Sample in air } \\
\text { (grams) }\end{array}$ & $\begin{array}{l}\text { D } \\
\text { Weight of Bags } \\
\text { and Sample in } \\
\text { Water (grams) }\end{array}$ & $\begin{array}{c}E \\
(A+B+C)-D \\
\text { Total Volume }\end{array}$ & $\begin{array}{c}\mathrm{F} \\
\mathrm{A} / \mathrm{Vc}_{\mathrm{c}}+\mathrm{B} / \mathrm{Rc} \\
\text { Bag and } \\
\text { rubber sheet } \\
\text { Volume }\end{array}$ & $\begin{array}{c}\text { G } \\
\text { E-F } \\
\text { Sample } \\
\text { Volume }\end{array}$ & $\begin{array}{c}\mathrm{H} \\
\mathrm{C} / \mathrm{G} \\
\text { Density }\end{array}$ \\
\hline & & & & & & & & \\
\hline & & & & & & & & \\
\hline & & & & & & & & \\
\hline & & & & & & & & \\
\hline & & & & & & & & \\
\hline & & & & & & & & \\
\hline & & & & & & & & \\
\hline & & & & & & & & \\
\hline & & & & & & & & \\
\hline & & & & & & & & \\
\hline
\end{tabular}

$R_{c}=\quad \mathrm{g} / \mathrm{cm} 3$ (value written on rubber sheets) $V_{C}=0.903 \mathrm{~g} / \mathrm{cm}^{3}$

- Do not squeeze down on the bags, when spreading the sample.

- Before using the large bag, inspect the bag for holes or

\section{Important}

- To stop the possibility of air from getting into the bag, cut 1 (25mm) of the bag while at least 2" $(50 \mathrm{~mm})$ under the water.

stress points. Do not use damaged bags.

- Use a large water tank for conducting this test.

- Use all three white filler plates.

- Remove the sliding plate.

- Place rough side of the channeled bag down inside the

external bag.

- Immediately place the sealed sample in the water tank.

- Hold the bags open for 15 seconds to allow water to get in

- If you see a massive amount of bubbles (Like boiling water) coming out of the bag, repeat the test.

- While under water, place the sample in the weighing basket

- Use the alligar clip to kep the cut portion of the bag fret Use the alligator clip to floating out of water

Make sure the bag is not floating out of the water

- touching the sides and bottom of tank.

onsistently punctures bags.

Figure 5 - Data Chart for CoreLok $\mathrm{G}_{\mathrm{mm}}$ Test 
The basic concept behind these procedures is to find the volume of the sample with no air voids. The sample is first weighed dry, and then transferred to the "bucket" where water is added to cover the sample completely. At this point a vacuum is applied to the sample under the water to rid the sample of air. The sample is then weighed under water to acquire the volume of the sample. If the sample is contains absorbent aggregates the additional dry-back procedure is required, which takes between one to two hours. This additional procedure establishes the saturated surface dry weight, which is needed in the calculation:

$$
G_{m m}=\frac{A}{(B+D-E)}
$$

Where:

A= Oven Dry Weight

$\mathrm{B}=$ Saturated Surface Dry Weight

$\mathrm{D}=$ Weight of Container in Water

$\mathrm{E}=$ Weight of Container + Sample in Water.

\subsection{G $_{M M}$ INVESTIGATIONS USING THE CORELOK}

Several studies have investigated the use of the CoreLok to measure the bulk specific gravity of asphalt concrete. However, only two studies were found that investigated the use of the CoreLok for measuring the maximum theoretical specific gravity.

\subsubsection{Florida DOT}

The Florida Department of Transportation evaluated the CoreLok method for determining the bulk and maximum theoretical specific gravity of asphalt mixes and the bulk specific gravity of aggregates (Sholar et al., 2003). Only the $\mathrm{G}_{\mathrm{mm}}$ research is reviewed herein. Results using the CoreLok method were compared to results obtained with the FDOT test procedure FM 1-T 209, similar to AASHTO T209. The test set-up for FM 1-T-209, as shown on Figure 6, requires the weigh in air method and two specimens are required for each test result. In addition, FM 1-T-209 requires measuring the saturated surface dry mass of the sample using the dry-back process. An interesting note in the test method is that in the event that final surface dry mass of the specimen is less than the original mass of the dry specimen, then the original dry mass is used in lieu of the saturated surface dry mass for the calculation of $G_{m m}$. Sholar et al. noted that the 
dry weight is less than the saturated surface dry weight when the amount of material lost during the test is greater than the amount of water in the voids of the sample. This was an issue with the RAP mixes as a high percent of the RAP was uncoated and fines were lost when the water was drained from the sample.

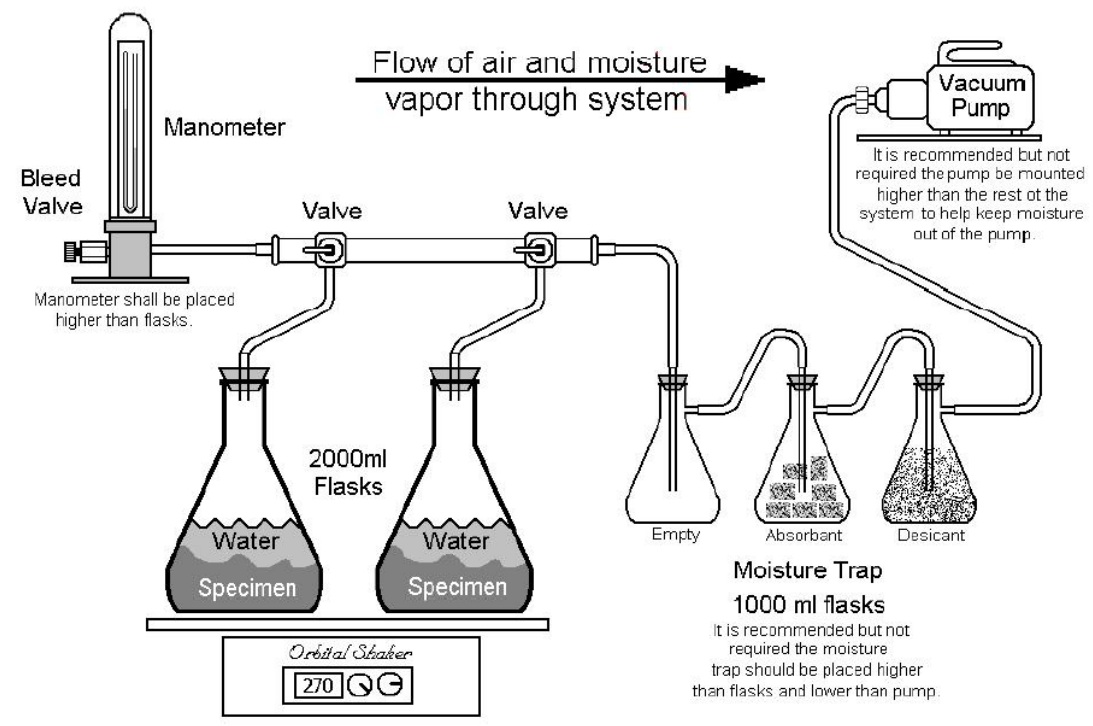

Figure 6- Test set up for FDOT test method FM 1-T209

Sholar et al. studied six different mixes with varying degrees of absorption, aggregate size, and material type. Ten replicate samples were tested for each material and test method. No slag was used in their experiment, but a highly absorptive limestone was used.

Table 1 presents the statistical analysis results of the FDOT study. Sholar et al. noted that the CoreLok $G_{m m}$ values were greater than the conventional results for all six sample types. The hypothesis of equal means was not rejected for the sample with the lowest absorption, G-1. The hypothesis of equal means was also not rejected for the RAP sample with percent water absorption of 1 to 2 percent. However, the p-value was 0.09 , indicating that if the confidence level for the statistical test was 90 percent rather than 95 percent, then the hypothesis of equal means would be rejected. Similarly, the t-test for limestone sample 2, LS-2, did not reject the hypothesis of equal means for a confidence level of 95 percent, but the p-value indicates the hypothesis would be rejected for a confidence level of less than 94 percent. The p-value for the highly absorptive aggregate, 
LS-4, indicates that there is virtually no chance that the results from the conventional and CoreLok methods are from the same population. The net conclusion was that the conventional and CoreLok methods produce different results for asphalt concrete made with moderate and highly absorptive aggregates.

Sholar et al. (2003) also compared the data they collected without using the dryback procedures and found only one mixture showed significant difference from the CoreLok test method. They concluded that using the CoreLok with high absorptive aggregates results in higher $\mathrm{G}_{\mathrm{mm}}$ values which would result in higher computed air voids in the mix. The reason for this was explained as the lack of a dry-back method with the CoreLok.

Table 1- FDOT t-test Results for $\mathrm{G}_{\mathrm{mm}}$ Test Data for the CoreLok and FM 1-T 209

\begin{tabular}{|l|c|c|c|c|c|c|}
\cline { 2 - 7 } \multicolumn{1}{c|}{} & \multicolumn{7}{c|}{ Mixture Designation } \\
\cline { 2 - 7 } \multicolumn{1}{c|}{} & G-1 & LS-1 & LS-2 & LS-3 & LS-4 & RAP \\
\hline t-statistic & 0.54 & 4.32 & 2.01 & 2.52 & 23.86 & 1.80 \\
\hline t-critical & 2.10 & 2.10 & 2.10 & 2.10 & 2.10 & 2.11 \\
\hline $\begin{array}{l}\text { Significantly } \\
\text { Different? }\end{array}$ & No & Yes & No & Yes & Yes & No \\
\hline p-value & 0.59 & $4.12 \mathrm{E}-04$ & 0.06 & 0.02 & $4.47 \mathrm{E}-15$ & 0.09 \\
\hline $\begin{array}{l}\text { Percent water } \\
\text { absorption }\end{array}$ & $<1$ & 2 to 3 & 2 to 3 & 2 to 3 & 5 to 6 & 1 to 2 \\
\hline Difference & 0.001 & 0.011 & 0.002 & 0.004 & 0.033 & 0.002 \\
\hline
\end{tabular}

${ }^{*}$ CoreLok - Conventional

\subsubsection{University of Cincinnati}

Rajagopal and Crago (2007) evaluated the CoreLok relative to AASHTO test methods for both the bulk and maximum theoretical specific gravity. Only the $G_{m m}$ results are reviewed in the following. The experimental design included a range of mix types and aggregate sources, including four mixes with slag aggregate, resulting in 33 samples. Although not explicitly stated, the paired t-test analysis method used to evaluate the data suggests that each sample was tested with both the AASHTO and CoreLok methods. The test results are presented in Table 2. The mean $G_{m m}$ values for the test methods are equal with a value of 2.444. As would be expected the t-test did not identify a significant difference between the two methods. A regression analysis found a 
strong correlation between the two methods, with an r value of 0.53 . However, the slope of the regression was 0.92 which indicates the results from the CoreLok are lower than T-209 for low $G_{m m}$ values and vice versa for high $G_{m m}$ values. 
Table 2 - Comparison Data for AASHTO T-209 and CoreLok (Rajagopal and Crago 2007)

\begin{tabular}{|l|c|c|}
\hline Mix type & AASHTO T-209 & CoreLok \\
\hline Dense Grade Heavy Gravel & 2.447 & 2.393 \\
\hline Dense Grade Heavy Lime Stone & 2.443 & 2.455 \\
\hline Dense Grade Heavy Gravel & 2.445 & 2.443 \\
\hline Dense Grade Heavy Lime Stone & 2.500 & 2.529 \\
\hline Dense Grade Heavy Gravel & 2.422 & 2.410 \\
\hline Dense Grade Heavy Lime Stone & 2.504 & 2.530 \\
\hline Dense Grade Heavy Lime Stone & 2.486 & 2.461 \\
\hline Dense Grade Medium Gravel & 2.447 & 2.446 \\
\hline Dense Grade Medium Lime Stone & 2.491 & 2.508 \\
\hline Dense Grade Medium Gravel & 2.466 & 2.459 \\
\hline Dense Grade Medium Lime Stone & 2.450 & 2.457 \\
\hline Superpave Medium Slag & 2.351 & 2.308 \\
\hline Superpave Medium Lime Stone & 2.457 & 2.321 \\
\hline Superpave Medium Slag & 2.379 & 2.409 \\
\hline Superpave Medium Lime Stone & 2.475 & 2.478 \\
\hline Superpave Medium Slag & 2.354 & 2.389 \\
\hline Superpave Medium Lime Stone & 2.468 & 2.483 \\
\hline Superpave Heavy Lime Stone & 2.465 & 2.511 \\
\hline Superpave Heavy Slag & 2.437 & 2.410 \\
\hline Superpave Heavy Lime Stone & 2.512 & 2.509 \\
\hline Superpave Heavy Slag & 2.411 & 2.379 \\
\hline Superpave Heavy Lime Stone & 2.462 & 2.457 \\
\hline SMA Heavy Lime Stone & 2.452 & 2.451 \\
\hline SMA Heavy Lime Stone & 2.360 & 2.442 \\
\hline SMA Heavy Lime Stone & 2.384 & 2.387 \\
\hline SMA Heavy Lime Stone & 2.427 & 2.398 \\
\hline 302 Heavy Gravel & 2.511 & 2.460 \\
\hline 302 Medium Gravel & 2.475 & 2.481 \\
\hline 302 Medium Lime & 2.498 & 2.561 \\
\hline 302 Medium Slag & 2.444 & 2.483 \\
\hline SMA Heavy Lime Stone & 2.400 & 2.419 \\
\hline SMA Heavy Lime Stone & 2.408 & 2.404 \\
\hline SMA Heavy Gravel & 2.429 & 2.427 \\
\hline \hline Averages & & 2.444 \\
\hline
\end{tabular}




\section{CHAPTER 3 RESEARCH METHODOLOGY}

\subsection{INTRODUCTION}

The experimental plan was designed to evaluate differences in maximum theoretical specific gravity between lab produced and plant produced mixes with a blend of slag and limestone aggregates. Maximum theoretical gravity was measured using the conventional (Rice), dry-back, and CoreLok methods. The materials for this experiment were provided by West Virginia Paving. The asphalt cement was a PG 64-22. The three types of stones were limestone sand, a No. 8 slag, and a No. 8 limestone.

\subsection{EXPERIMENTAL DESIGN}

The factors and levels for the experiment are shown in Table 3. Three levels of slag content were used as increasing the slag content should increase the chance for residual moisture. The Superpave mix design method, using 80 gyrations for $\mathrm{N}_{\text {design }}$, was used to establish the design binder content for each blend. Samples were prepared at the design binder content and at the design binder content plus 0.5 percent. The higher binder content was included in the experiment to test if better sealing of the voids in the slag, due to a greater binder film thickness, would alter the volumetric properties of the mix. One of the issues when comparing lab prepared mixes versus plant mixes is the potential for residual moisture on the aggregates of the plant mixture. An attempt was made to simulate this by saturating the aggregates and then only allowing them to dry until the target residual moisture content remained before blending the mix. Another issue for this comparison is the lack of a conditioning time for the plant samples; therefore two conditioning times were tested. Table 4 shows the data summary sheet. Table 3 - Experimental Factors/Levels

\begin{tabular}{|l|l|}
\hline Slag Content & $12 \%, 27 \%, 42 \%$ \\
\hline Asphalt Content & Design(dependent on the Mix), Design $+0.5 \%$ \\
\hline Moisture Content & $0 \% \mathrm{MC}, 2 \% \mathrm{MC}$ \\
\hline Conditioning Time & Design(2 hours), Zero conditioning time \\
\hline Test Method & Rice, Rice with additional Dry-Back, CoreLok \\
\hline
\end{tabular}


Table 4 - Data Table

\begin{tabular}{|c|c|c|c|c|c|c|c|c|}
\hline & & & \multicolumn{6}{|c|}{$\mathrm{Gmm}$} \\
\hline & & & \multicolumn{6}{|c|}{$\%$ slag } \\
\hline & & & \multicolumn{2}{|c|}{$12 \%$} & \multicolumn{2}{|c|}{$27 \%$} & \multicolumn{2}{|c|}{$42 \%$} \\
\hline \multirow{2}{*}{$\begin{array}{c}\% \\
\text { Asphalt }\end{array}$} & \multirow{2}{*}{$\begin{array}{c}\text { Test } \\
\text { Method }\end{array}$} & \multirow[b]{2}{*}{ Trial } & \multicolumn{6}{|c|}{$\%$ Moisture } \\
\hline & & & 0 & 2 & 0 & 2 & 0 & 2 \\
\hline \multirow{6}{*}{$0.50 \%$} & Comole & 1 & & & & & & \\
\hline & CUICLUK & 2 & & & & & & \\
\hline & & 1 & & & & & & \\
\hline & RIce & 2 & & & & & & \\
\hline & Dry- & 1 & & & & & & \\
\hline & Back & 2 & & & & & & \\
\hline \multirow{6}{*}{ Design } & & 1 & & & & & & \\
\hline & COIELOK & 2 & & & & & & \\
\hline & & 1 & & & & & & \\
\hline & Rice & 2 & & & & & & \\
\hline & Dry- & 1 & & & & & & \\
\hline & Back & 2 & & & & & & \\
\hline \multirow{6}{*}{$\begin{array}{c}\text { Zero } \\
\text { Condition } \\
\text { Time }\end{array}$} & & 1 & & & & & & \\
\hline & CUICLUK & 2 & & & & & & \\
\hline & & 1 & & & & & & \\
\hline & Rice & 2 & & & & & & \\
\hline & Dry- & 1 & & & & & & \\
\hline & Back & 2 & & & & & & \\
\hline
\end{tabular}

\subsection{MATERIAL}

\subsubsection{Asphalt Cement}

The asphalt binder used was supplied by WV Paving. The binder is a performance grade, (PG) 64-22, sourced from Shelly Liquid Division located in Gallipolis, Ohio. This batch of binder had a mixing temperature range of 152 to $165^{\circ} \mathrm{C}$ and a compaction temperature of 140 to $146^{\circ} \mathrm{C}$. The binder had a specific gravity of 1.021 . 


\subsubsection{Aggregate Properties}

Three aggregates were used for the entire project; they were limestone sand (L/S sand), a No. 8 limestone (L/S), and a No. 8 slag stone. All the aggregates were gathered from the Dunbar, WV plant owned by West Virginia Paving. Table 5shows the producer, the bulk specific gravity $\left(\mathrm{G}_{\mathrm{sb}}\right)$, and the apparent specific gravity $\left(\mathrm{G}_{\mathrm{sa}}\right)$ of each aggregate. The slag was an air cooled blast furnace steel slag.

Table 5 - Aggregate Properties

\begin{tabular}{|c|c|c|c|c|}
\hline Material & Producer & Location & $\mathrm{G}_{\mathrm{sb}}$ & $\mathrm{G}_{\mathrm{sa}}$ \\
\hline L/S Sand & Mulzer & Cape Sandy & 2.619 & 2.736 \\
\hline L/S \#8 & Carmeuse & Maysville & 2.643 & 2.735 \\
\hline Slag \#8 & Mountain & Greenup & 2.553 & 2.664 \\
\hline
\end{tabular}

\subsubsection{Aggregate Preparation}

The aggregates were air dried. Three samples of each aggregate type were randomly sampled for a washed gradation analysis. The gradations for each aggregate are shown in Table 6. The remaining materials was washed to remove the mineral filler, dried, sieved and each size-type combination was stored in a separate bin. Bag house fines were used in the mixtures as a substitute for the mineral filler lost when washing.

Table 6 - Aggregate Gradations

\begin{tabular}{|c|c|c|c|c|}
\hline $\begin{array}{c}\text { Sieve } \\
\text { Size (US) }\end{array}$ & $\begin{array}{c}\text { Sieve } \\
\text { Size }(\mathrm{mm})\end{array}$ & \#8 LS & \#8 Slag & LS Sand \\
\hline 1 & 25 & 100.0 & 100.0 & 100.0 \\
\hline $3 / 4$ & 19 & 100.0 & 100.0 & 100.0 \\
\hline $1 / 2$ & 12.5 & 100.0 & 100.0 & 100.0 \\
\hline $3 / 8$ & 9.5 & 88.3 & 90.5 & 100.00 \\
\hline$\# 4$ & 4.75 & 26.1 & 32.0 & 95.88 \\
\hline$\# 8$ & 2.36 & 8.3 & 14.7 & 73.80 \\
\hline$\# 16$ & 1.18 & 5.2 & 11.0 & 48.28 \\
\hline$\# 30$ & 0.6 & 4.2 & 8.0 & 31.70 \\
\hline$\# 50$ & 0.3 & 3.5 & 5.5 & 19.61 \\
\hline$\# 200$ & 0.075 & 2.9 & 3.5 & 7.41 \\
\hline
\end{tabular}




\subsection{DESIGN}

For the experiment, three mix designs were completed each with increasing amounts of slag. Each mix was designed according to AASHTO specifications. $\mathrm{G}_{\mathrm{mb}}$ samples were made and tested and the design binder content was selected for each mixture. The binder content for each mix was $6.2 \%$ for low slag, $6.5 \%$ for medium slag, and $7.0 \%$ for high slag content.

\subsubsection{Aggregate Blends}

The lower slag content mixture, 12 percent slag, represents a mix with less slag than used in production. The medium or 27 percent slag mix resembles a normal slag content production mix, and the higher 42 percent slag mixture resembles an extreme case where slag is the abundant material used. All three mixtures were $9.5 \mathrm{~mm}$ mixes and meet the gradation requirements, Table 7, for a Superpave mix design. Also listed in Table 7 are the combined gradations of each mixture along with the combined specific gravities. These mixes meet the Superpave definition of coarse mixes since the percent material passing the No. $8(2.36 \mathrm{~mm})$ sieve is less than the criteria of 47 percent. Mixtures 1, 2, and 3 are low slag, medium slag, and high slag content respectively. The combined gradations of the three mixtures are shown on Figure 7. Care was taken to eliminate the factor of gradation in this experiment. The gradations are relatively the same with the largest difference being 2 percent. This difference is between mix 1 and 3 on the No. $8(2.36 \mathrm{~mm})$ sieve.

When designing the blends, the slag content was increased in equal amounts of 15 percent to keep changes consistent. The percent of the limestone sand not changed between the mixtures. The amount of No. 8 limestone was decreased in proportion to the change in the change in the slag content. The resulting blends were:

Mix 1: 43\% L/S No. 8, 12\% Slag No. 8, and 45\% L/S sand

Mix 2: 28\% L/S No. 8, 27\% Slag No. 8, and 45\% L/S sand

Mix 3: 13\% L/S No. 8, 42\% Slag No. 8, and 45\% L/S sand 
Table 7 - Combined Mixture Gradations \& Properties

\begin{tabular}{|c|c|c|c|c|c|c|}
\hline & & \multicolumn{3}{|c|}{ Percent Passing } & \multicolumn{2}{c|}{ Control Points } \\
\hline $\begin{array}{c}\text { Sieve } \\
\text { Size (US) }\end{array}$ & $\begin{array}{c}\text { Sieve } \\
\text { Size (mm) }\end{array}$ & Mix 1 & Mix 2 & Mix 3 & Lower & Upper \\
\hline $1 / 2$ & 12.5 & 100.0 & 100.0 & 100.0 & 100 & \\
\hline $3 / 8$ & 9.5 & 93.8 & 94.2 & 94.5 & 90 & 100 \\
\hline 4 & 4.75 & 58.2 & 59.1 & 60.0 & - & 90 \\
\hline 8 & 2.36 & 38.5 & 39.5 & 40.5 & 32 & 67 \\
\hline 16 & 1.18 & 25.3 & 26.2 & 27.0 & - & - \\
\hline 30 & 0.6 & 17.0 & 17.6 & 18.2 & - & - \\
\hline 50 & 0.3 & 11.0 & 11.3 & 11.6 & - & - \\
\hline 200 & 0.075 & 5.0 & 5.1 & 5.2 & 2 & 10 \\
\hline & $\mathrm{G}_{\mathrm{sb}}$ & 2.621 & 2.607 & 2.594 & & \\
\hline & $\mathrm{G}_{\mathrm{sa}}$ & 2.727 & 2.716 & 2.705 & & \\
\hline
\end{tabular}

\subsection{SAMPLE CREATION}

Aggregate samples were weighed out according to a weigh table created for each mix. A sample of this table is show in Table 8.The aggregates were dried in the oven overnight prior to weighing to ensure there was no moisture in any of the stone. Once the sample was weighed, the trays along with a container of bag house fines, were placed in the oven the night prior to mixing and left at the mixing temperature. Also, the night before the bucket, paddle, binder, and tools were placed in a second oven with a timer to start so that everything was at the mix temperature prior to blending.

Once everything was at the correct mixing temperature, the aggregate was added to the mixing bucket. A crater was made in the aggregates for the asphalt binder. The required amount of binder was weighed into the aggregates. The sample was then mixed with a bucket mixer to ensure adequate coating of all the aggregates. After mixing, the samples were placed in the oven at compaction temperature for two hours with stirring after one hour. After conditioning, the sample was emptied from the pans onto a stainless steel table, spread out and allowed to cool. This procedure is for the basic mixtures, there were modifications to this procedure for the two conditioning scenarios in the experiment. These modifications are explained in the following sections. 


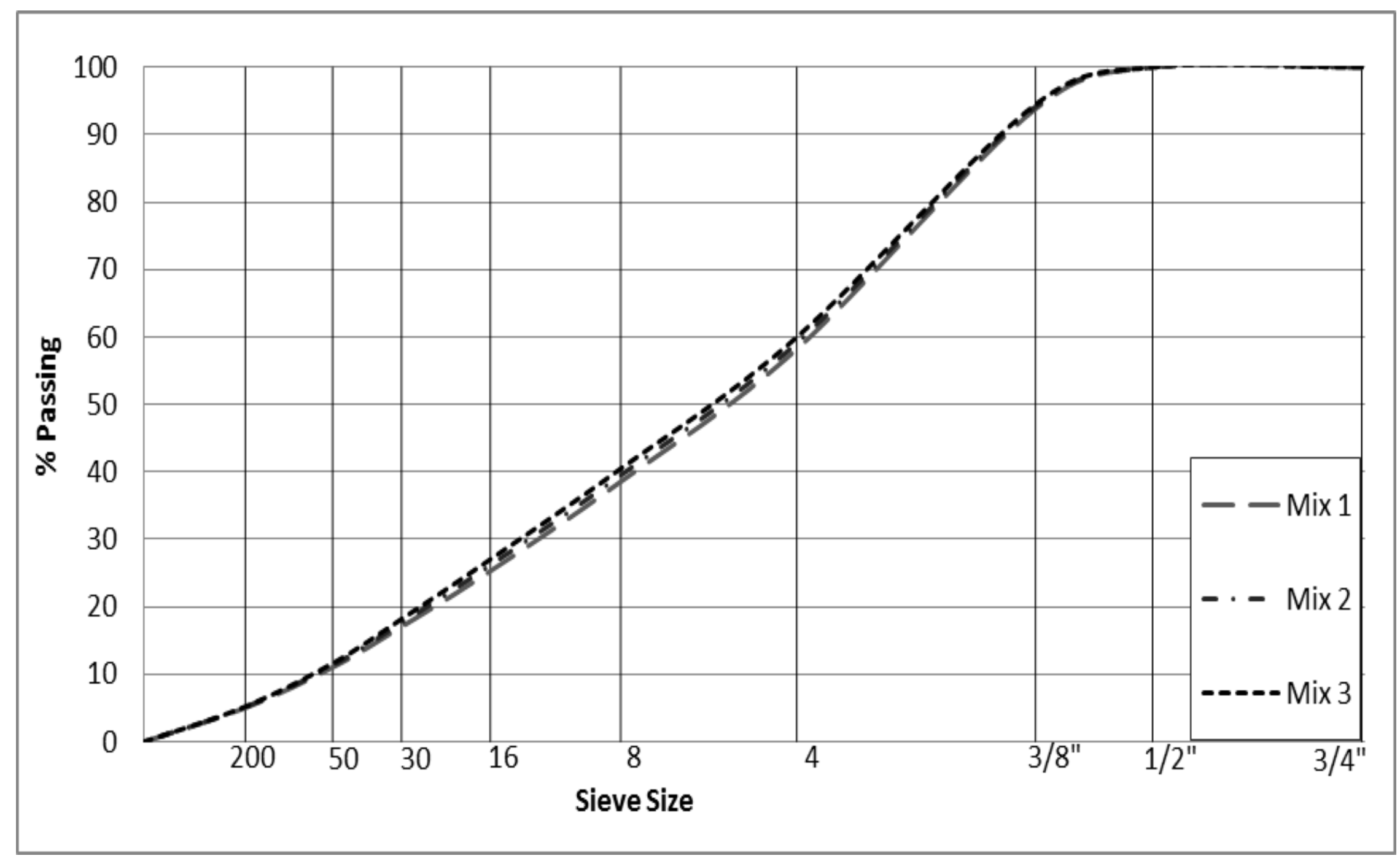

Figure 7 - Combined Mixture Gradations 
Table 8-Example Aggregate Weigh-out Table

\begin{tabular}{|c|c|c|c|c|c|c|c|c|c|}
\hline & & & 13 & $\begin{array}{l}\text { Design } \\
3-42-45+0\end{array}$ & $\begin{array}{l}\mathrm{AC} \\
0.0 \% \mathrm{MC}\end{array}$ & & & & \\
\hline & & Gmb & & Gmm & & & P_b Total & $7.00 \%$ & \\
\hline & - & g & $1,515.0$ & $\mathrm{~g}$ & Total S & Sample V & Weight & $3,030.0$ & $\mathrm{~g}$ \\
\hline & Percent $\mathrm{S}$ & Stone P S S & $93.0 \%$ & & $\% \mathrm{~V}$ & Virgin Binc & der & $7.0 \%$ & \\
\hline & Weight of & Stone $\mathrm{W} \_\mathrm{s}$ & $2,817.9$ & & Weight & ti of Binde & er W_b & 212.1 & $\mathrm{~g}$ \\
\hline & Stockpile & L/S \#8 & $g$ & Stockpile & Slag \#8 & $\mathrm{g}$ & Stockpile & L/S Man & g \\
\hline & Blend & $13.0 \%$ & 366.3 & Blend & $42.0 \%$ & 1183.5 & Blend & $45.0 \%$ & 1268.1 \\
\hline Sieve Size & $\%$ Passing & $\%$ Retained & Weight & $\%$ Passing & $\%$ Retained & Weight & $\%$ Passing & $\%$ Retained & Weight \\
\hline 25 & 100.0 & 0.0 & 0.0 & 100.0 & 0.0 & 0.0 & 100.0 & 0.0 & 0.0 \\
\hline 19 & 100.0 & 0.0 & 0.0 & 100.0 & 0.0 & 0.0 & 100.0 & 0.0 & 0.0 \\
\hline 12.5 & 100.0 & 0.0 & 0.0 & 100.0 & 0.0 & 0.0 & 100.0 & 0.0 & 0.0 \\
\hline 9.5 & 88.3 & 11.7 & 42.9 & 90.5 & 9.5 & 112.4 & 100.00 & 0.0 & 0.0 \\
\hline 4.75 & 26.1 & 62.2 & 227.9 & 32.0 & 58.5 & 692.3 & 95.88 & 4.1 & 52.2 \\
\hline 2.36 & 8.3 & 17.8 & 65.2 & 15 & 17.0 & 201.2 & 73.80 & 22.1 & 280.0 \\
\hline 1.18 & 5.2 & 3.0 & 11.2 & 12 & 3.0 & 35.5 & 48.28 & 25.5 & 323.6 \\
\hline 0.06 & 4.2 & 1.0 & 3.8 & 10.5 & 1.5 & 17.8 & 31.70 & 16.6 & 210.3 \\
\hline 0.03 & 3.5 & 0.6 & 2.4 & 8 & 2.5 & 29.6 & 19.61 & 12.1 & 153.2 \\
\hline 0.075 & 2.9 & 0.6 & 2.3 & 3 & 5.0 & 59.2 & 7.41 & 12.2 & 154.8 \\
\hline Pan & 0.0 & 2.9 & 10.7 & 0.0 & 3.0 & 35.5 & 0.00 & 7.4 & 93.9 \\
\hline Total & & 100.0 & 366.4 & & 100.0 & 1183.5 & & 100.0 & 1268.0 \\
\hline
\end{tabular}

\subsubsection{Additional Procedures for Water Addition}

The main conditioning scenario for this experiment involves the remnants of water inside the slag aggregate when it is in the mixture. After mixing, the water in the slag begins to evaporate and the water vapor may pierce the asphalt coating, which can create small voids for water to get in during the testing process. For the samples that include residual water in the aggregates the procedure was modified to include: saturation, drying, and mixing.

\subsubsection{Saturation}

During the weigh out procedure the slag was separated at the No. 8 sieve. The material that was retained above the No. 8 sieve was the only material that was saturated with water. The finer material was added to the mixture completely dry. This was done because in a blast furnace the finer material would be able to heat up faster than the larger material, therefore the moisture is most likely able to evaporate quicker. This was also 
done so that finer material would not be lost when excess water was emptied from the tray.

The material retained on the No. 8 sieve was saturated by placing it in a container, covering with water, and vibrating while a vacuum was applied. The vacuum was set to $55 \pm 2.5 \mathrm{mmHg}$. The sample was subjected to the vacuum for 30 minutes and then submerged under water for an additional $24 \pm 1$ hour. The quantity of aggregate for the high, 42 percent, slag mix was too large to fit in the container, so it was split in half and each half underwent the vacuum process one after the other and then were submerged together for the 24 hour period.

\subsubsection{Drying}

The following morning the sample was drained of the excess water. This was done over a No. 200 sieve to avoid the loss of material. Afterwards the sample was placed in the oven to heat up and dry to the desired moisture content. The saturated aggregate was placed in a separate oven from the other materials and bucket. This was done to limit the heat loss of the other material from opening the oven door to many times. After the slag had been in the oven for a short time, the weight was taken to see if it had reached the weight needed for the required two percent moisture content. This two percent moisture is based on the entire weight of the slag portion of the mixture. For example if there was 1000 grams of slag in the mix, 20 grams of water was required to be retained in the material retained above the No. $8(2.36 \mathrm{~mm})$ sieve. The slag was stirred multiple times during the drying process to resemble what happens to the rocks when they go through the plant dryer.

\subsubsection{Mixing}

Once the slag had reached the required 2\% moisture content it was removed from the oven and placed in the bucket that was already at the correct mixing temperature. To avoid further moisture loss, the slag was always placed in the bucket first and quickly followed by the sand, dust, and limestone. After all the materials were in the bucket it was weighed and recorded. Then the sample was weighed again after mixing was completed to see how much water had evaporated during the mixing process. 


\subsubsection{Additional Procedures for Zero Conditioning Time}

The other main conditioning scenario investigated was zero conditioning time. When a sample is taken from the plant it is immediately brought to the lab and tested. Since this occurs, a shorter conditioning time for lab created samples was examined. Zero conditioning time was chosen to show the extreme case of the lack of conditioning in plant produced samples. The only change to the procedure was that the sample was taken straight from the mixing bucket and spread out on the stainless steel table top. The samples that had an addition of water to the mix followed the same mixing procedures that were listed in the above section, but the two hour conditioning time was again eliminated.

\subsection{SAMPLE TESTING}

After the samples were mixed and conditioned (if required) they were spread on the table for cooling. While still lukewarm and pliable, the samples were broken apart into small pieces and stirred to make sure no large clumps formed. The sample was stirred again after a few minutes and then allowed to cool to near room temperature.

Once the sample cooled, it was gathered into a pile and split into quarters, as per the AASHTO T-268's sampling method. The entire sample consisted of two test samples. Each of these test samples were originally set to weigh 1515 grams. The required sample size for a Rice test is at least 1500 grams for a $9.5 \mathrm{~mm}$ mix, and the CoreLok has a maximum sample size of 2000 grams. Each sample was then tested with each test method. The data collected during the dry-back procedure was used for the conventional Rice method, simply ignoring the weight recorded after the additional dryback.

\subsubsection{Test Methods}

Three test methods were used for this investigation: the conventional AASHTO T-209 Rice method, the T-209 with additional Dry-Back procedure, and the CoreLok. Each test was completed using the procedures listed in the respective manuals; are described in Chapter 2. In order to complete the two test samples for the dry-back procedure, a drying rack was fabricated that held two No. 30 sieves held at an angle for 
each material. A fan was then placed behind each of the sieves and blew air up through the bottom of the sieves. Figure 8 shows the drying rack.

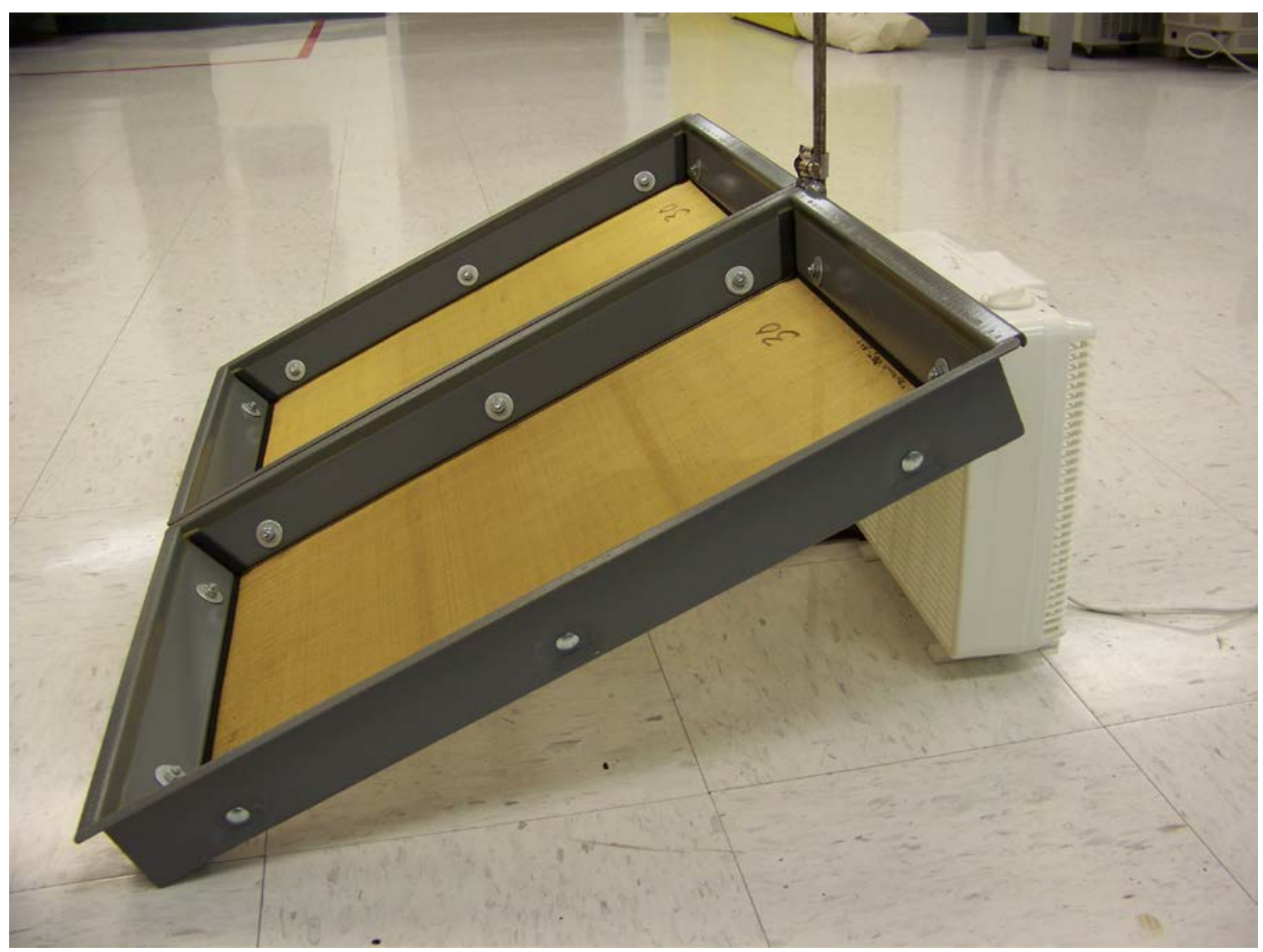

Figure 8 - Drying Rack 


\section{CHAPTER 4 RESULTS AND ANALYSIS}

\subsection{INTRODUCTION}

A total of 18 mixes were tested for determining maximum theoretical specific gravity use CoreLok, and AASHTO's T-209 method with and without the dry-back procedures. The factors and levels of the experiment are shown in Table 9. Two samples were made and used for each testing device, totaling 36 samples and 108 tests. Each group of results was given an acronym to describe its mixture properties and test procedure, as defined in Table 10. For example LPCM means Low slag content (12 percent), Plus an additional half percent asphalt, and CoreLok test method, and two percent Moisture.

Table 9 - Experimental Factors and Levels

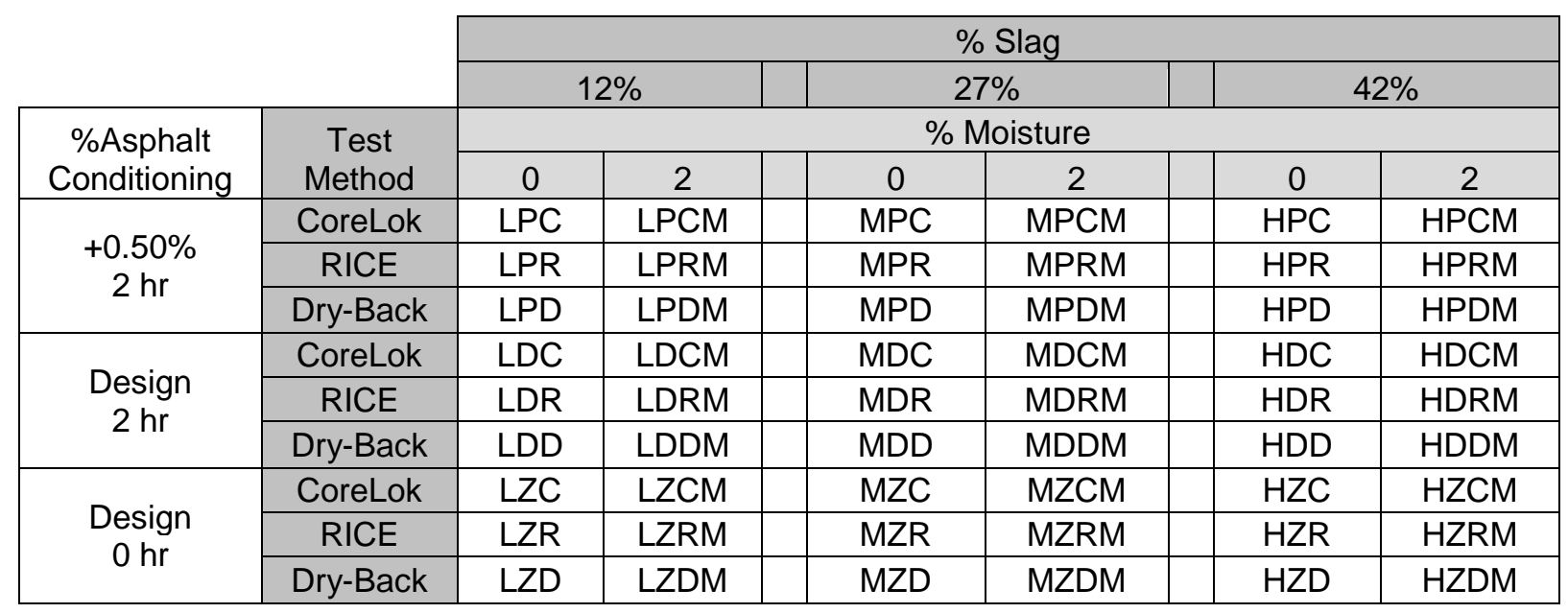

Table 10 - Description of Acronyms

\begin{tabular}{|l|l|}
\hline \multicolumn{2}{|c|}{ First Letter } \\
\hline L & Low-12\% SLAG \\
\hline M & Medium-27\% SLAG \\
\hline H & High-42\% SLAG \\
\hline
\end{tabular}

\begin{tabular}{|l|l|}
\hline \multicolumn{2}{|c|}{ Third Letter } \\
\hline C & CORELOK \\
\hline R & RICE METHOD \\
\hline D & DRYBACK \\
\hline
\end{tabular}

\begin{tabular}{|l|l|}
\hline \multicolumn{2}{|c|}{ Second Letter } \\
\hline P & Plus additional 0.5\% asphalt \\
\hline D & Design AC \\
\hline$Z$ & Zero curing time \\
\hline
\end{tabular}

\begin{tabular}{|l|l|}
\hline \multicolumn{2}{|c|}{ Forth Letter } \\
\hline$M$ & Residual Moisture \\
\hline & \\
\hline & \\
\hline
\end{tabular}




\subsection{RESULTS}

The $\mathrm{G}_{\mathrm{mm}}$ data are presented in Table 11 and Table 12 shows the means and standard deviations of the $G_{m m}$ values. The raw data used for the following analysis is presented in the Appendix.

Table 11 - $\mathrm{G}_{\mathrm{mm}}$ Test Results

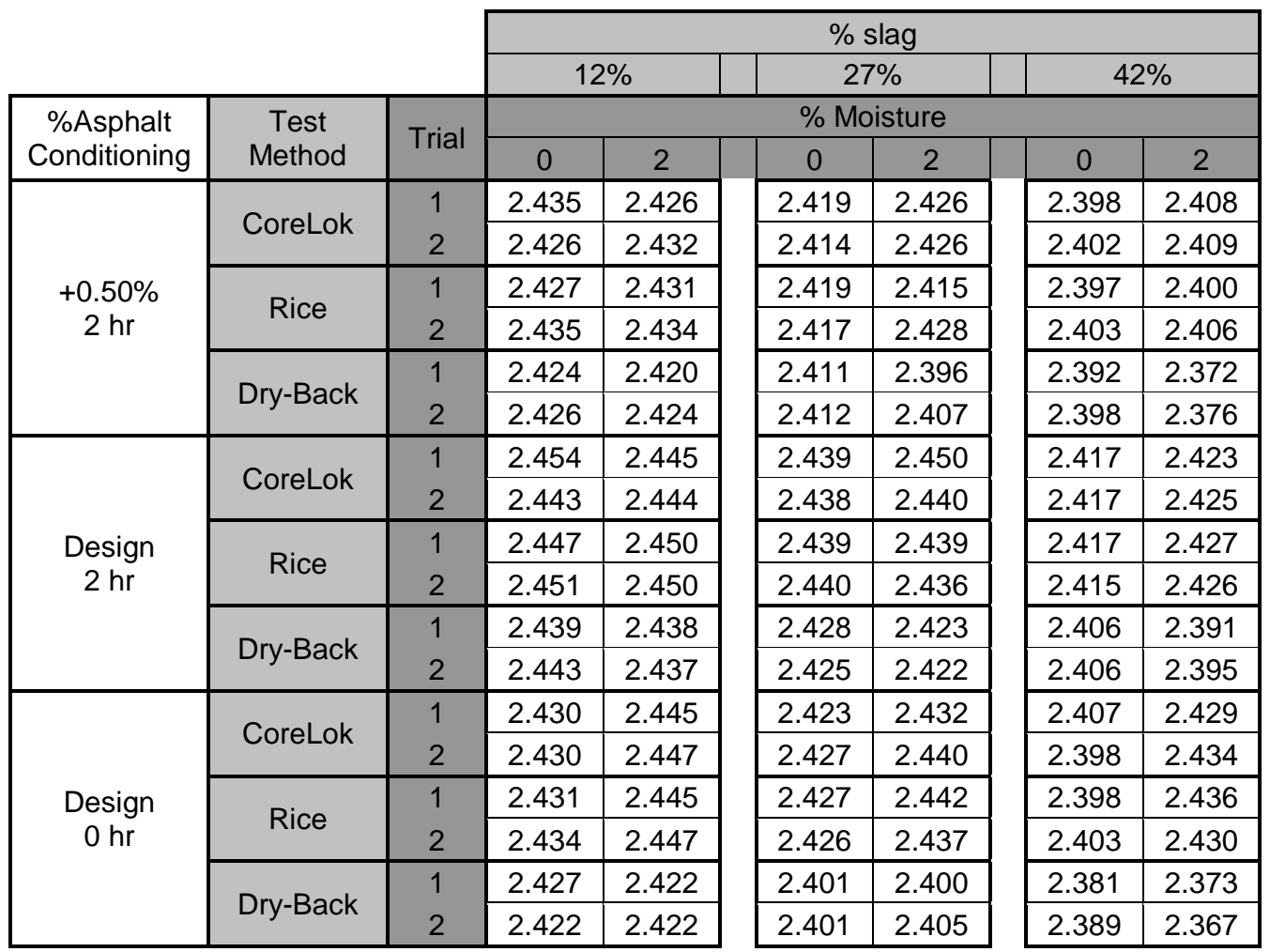

Table 12 - $\mathrm{G}_{\mathrm{mm}}$ Means and Standard Deviations

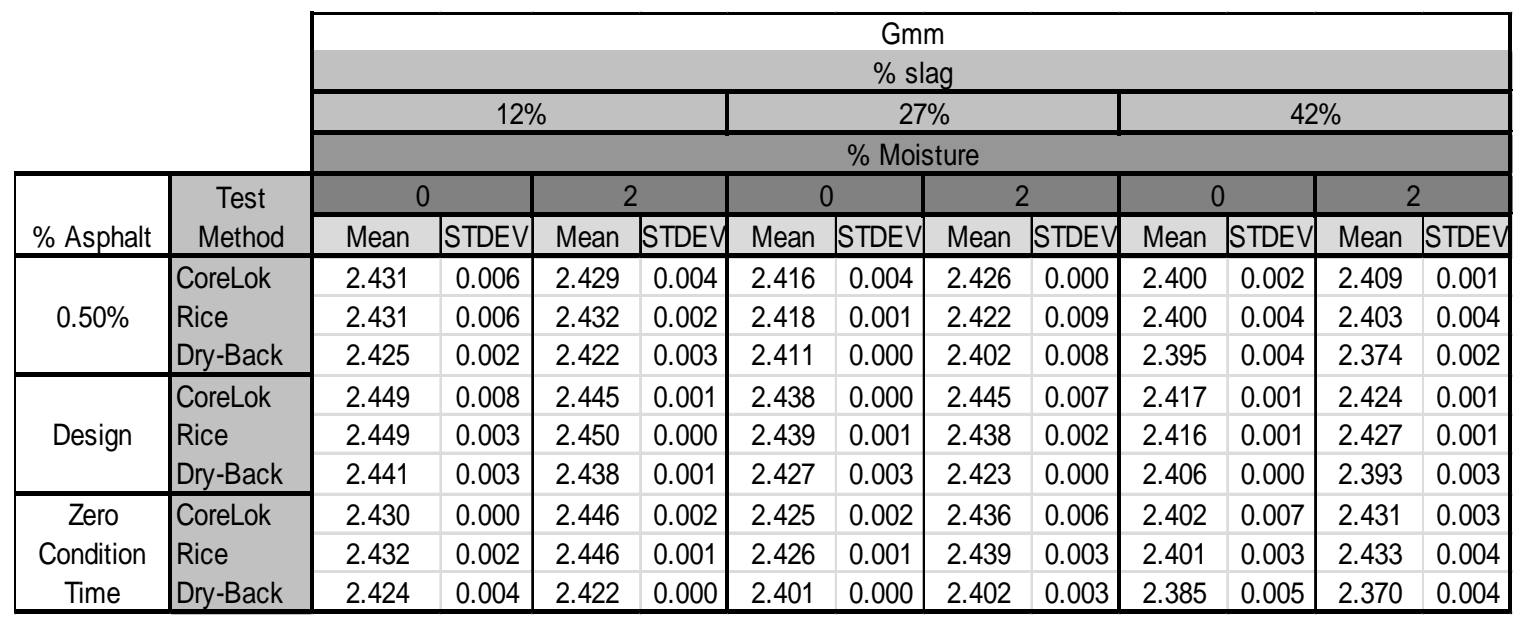




\subsection{1 $\mathrm{G}_{\mathrm{mm}}$ Analysis of Test Methods}

If the three test methods are equally effective at measuring $G_{m m}$ then they should produce equal results. The t-test, used to compare means when fewer than 30 observations are available, evaluates if the null hypothesis, $\mathrm{H}_{\mathrm{n}}$, of equal means can be rejected for a certain confidence level. The alternative hypothesis, $\mathrm{H}_{0}$, is for non-equal means. The decision provided by the t-test is either to reject the null hypothesis or there is insufficient evidence to reject the null hypotheses. The t-test does not determine if the null hypothesis should be accepted, but this is the implication of not rejecting the null hypothesis. A confidence level of $95 \%$ was used for the decision for all of the following statistical analysis. An inherent assumption of the t-test is the data are normally distributed. The following analysis was performed on the average of two replicate observations. In this situation the central limits theorem applies which justifies the normality assumption.

\subsubsection{Rice versus CoreLok}

Table 13 presents the results of the t-test's comparing the Rice versus CoreLok, Rice versus Dry Back and CoreLok versus Dry Back. The analysis was performed with Excel assuming equal variance of the populations. The comparison of the Rice and the CoreLok methods indicates the null hypothesis cannot be rejected with the inference that the results of the two methods are the same. The comparisons of the Rice versus Dry Back and CoreLok versus Dry back indicates the null hypothesis of equal means can be rejected with the inference that the test methods produce difference results with a confidence level of 95\%.

The t-test does not provide any in site into the relationships between the data sets. To further evaluate the data a line of equality was used. When two test methods should produce the same results, a plot of the results of one method versus the other should produce a straight line with an intercept at the origin and a slope of one. A linear regression line fit to the data would have the equation:

$$
\mathrm{Y}=\mathrm{aX}+\mathrm{b} \pm \mathrm{e}
$$


Where $a$ is the slope of the line and $b$ is the intercept and e is the standard error. The coefficients can then be tested to determine if $\mathrm{a}=1$ and $\mathrm{b}=0$ for a given confidence level.

Table 13 - t-test comparisons of test methods

\begin{tabular}{|c|c|c|c|c|c|c|}
\hline & Rice & CoreLok & Dry-Back & CoreLok & Dry-Back & Rice \\
\hline Mean & 2.428 & 2.428 & 2.409 & 2.428 & 2.409 & 2.428 \\
\hline Variance & 0.000241 & 0.000214 & 0.000409 & 0.000214 & 0.000409 & 0.000241 \\
\hline Observations & 36 & 36 & 36 & 36 & 36 & 36 \\
\hline Pooled Variance & 0.000228 & & 0.000312 & & 0.000325 & \\
\hline $\begin{array}{l}\text { Hypothesized } \\
\text { Mean Difference }\end{array}$ & 0 & & 0 & & 0 & \\
\hline df & 70 & & 70 & & 70 & \\
\hline t Stat & 0.044 & & -4.501 & & -4.444 & \\
\hline $\mathrm{P}(\mathrm{T}<=\mathrm{t})$ one-tail & 0.482 & & 0.000 & & 0.000 & \\
\hline t Critical one-tail & 1.667 & & 1.667 & & 1.667 & \\
\hline $\mathrm{P}(\mathrm{T}<=\mathrm{t})$ two-tail & 0.965 & & 0.000 & & 0.000 & \\
\hline t Critical two-tail & 1.994 & & 1.994 & & 1.994 & \\
\hline Decision & \multicolumn{2}{|c|}{ cannot reject $\mathrm{Hn}$} & \multicolumn{2}{|c|}{ reject $\mathrm{Hn}$} & \multicolumn{2}{|c|}{ reject $\mathrm{Hn}$} \\
\hline
\end{tabular}

Figure 9 presents the line of equality for the Rice versus CoreLok comparison. The data appear very similar as would be expected from the results of the t-test. A trend line was fit to the data. The $\mathrm{R}^{2}$ is 0.97 , which indicates almost all of the variability in the data is explained by the regression equation. The slope of 1.05 is very close to the expected value of 1.0 and the intercept of -0.12 is close to the expected value of 0.0 .

The Excel trend line analysis does not provide the statistics needed to evaluate the statistical significance of the regression coefficients. The regression feature of Excel provides these statistics. Table 14 presents the regression analysis for the Rice versus CoreLok comparison. Since the trend line equation on the graph provides the coefficients, the only information needed from the regression table is the p-values for the intercept and slope. Since the p-value of the intercept, 0.380 , is greater than 0.05 , the null hypothesis that the intercept is equal to 0 cannot be rejected, inferring that the computed intercept is not statistically significantly different from 0 . The p-value Excel computes 


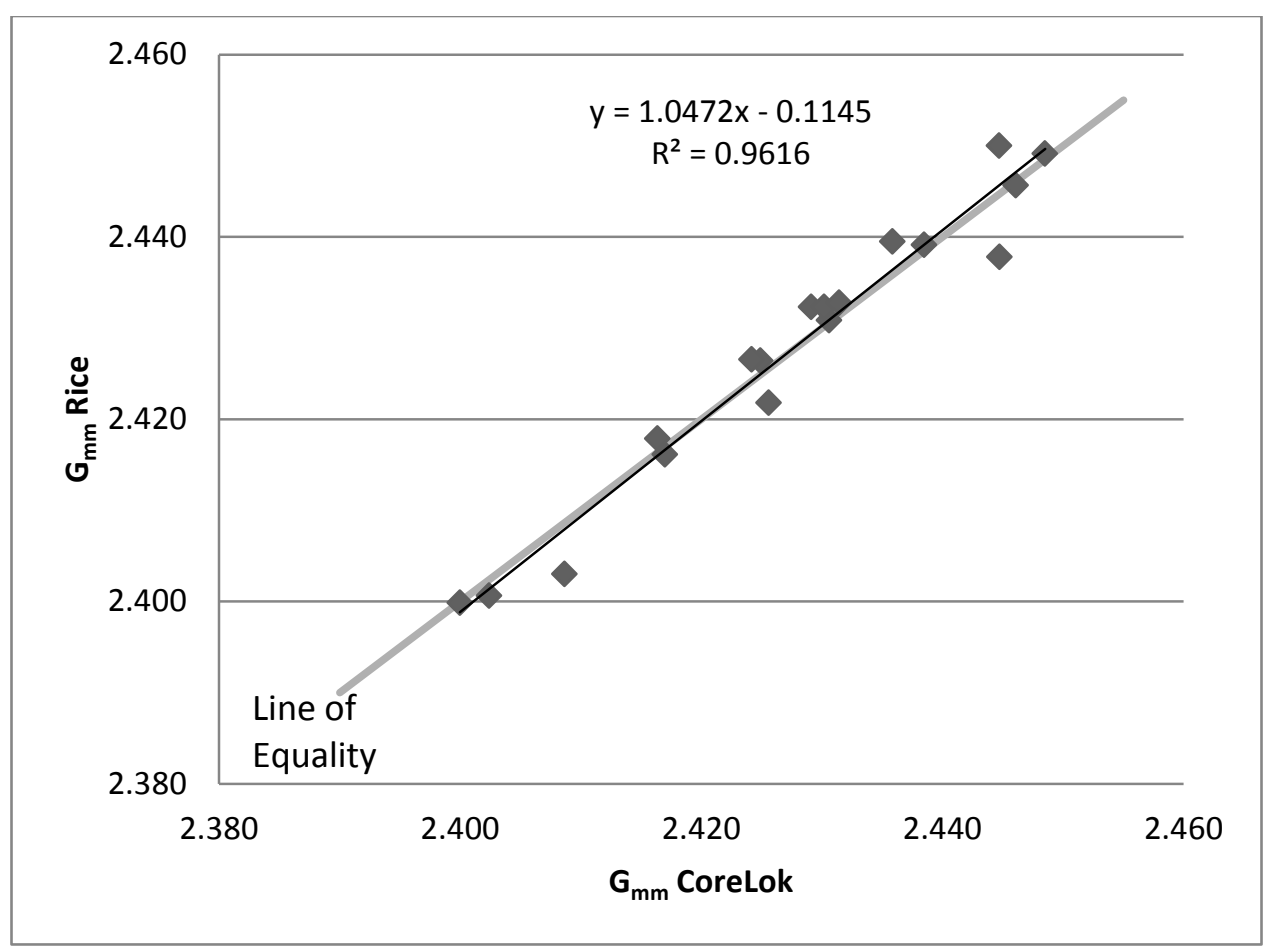

Figure 9 - Line of Equality Chart for Rice versus CoreLok $\mathrm{G}_{\mathrm{mm}}$ Results.

for the intercept is for a null hypothesis of 0 . A supplemental calculation is required to test for a null hypothesis of the slope equal to 1 . The adjusted t-value for a slope of 1 is computed as:

$$
\mathrm{t}=(\text { Slope }-1) /(\text { Standard Error })
$$

Then the p-value is computed using the TDIST function using the adjusted $\mathrm{t}$-value, the residual degrees of freedom and the two tail assumption as the arguments for the function. In Table 14 the computed p-value is 0.380 . Since this value is greater than 0.05 the null hypothesis cannot be rejected, inferring that the regression slope is not statistically different from 1.0.

The line of equality analysis results, shown in Table 14 agree with the t-value analysis in that the conclusion is the Rice and CoreLok methods produce results that are not significantly different. The advantage of the line of equality approach is that the relationship between the data is shown. In addition, the line of equality approach has the ability to determine if there is a bias between the two methods as the intercept of the regression equation. 
Table 14 - Results of Regression Analysis for Rice versus CoreLok

\begin{tabular}{|c|c|c|c|c|}
\hline \multicolumn{2}{|c|}{ Regression Statistics } & & & \\
\hline Multiple R & 0.981 & & & \\
\hline R Square & 0.962 & & & \\
\hline Adjusted R Square & 0.959 & & & \\
\hline Standard Error & 0.003 & & & \\
\hline Observations & 18 & & & \\
\hline \multicolumn{5}{|l|}{ ANOVA } \\
\hline & $d f$ & SS & MS & $F$ \\
\hline Regression & 1 & 0.004 & 0.004 & 401.164 \\
\hline Residual & 16 & 0.000 & 0.000 & \\
\hline \multirow[t]{2}{*}{ Total } & 17 & 0.004 & & \\
\hline & Coefficients & Std Error & t Stat & P-value \\
\hline Intercept & -0.114 & 0.127 & -0.902 & 0.380 \\
\hline$X$ Variable 1 & 1.047 & 0.052 & 20.029 & 0.000 \\
\hline tails & 2 & & Decision & \\
\hline$t$ for $\mathrm{Hn}=1$ & 0.903 & & Intercept & cannot reject Hn \\
\hline$p$-value for $H n=1$ & 0.380 & & Slope & cannot reject $\mathrm{Hn}$ \\
\hline
\end{tabular}

\subsubsection{Dry Back versus Rice}

Figure 10 presents the line of equality graph for the Dry Back versus Rice test methods. The p-value for the slope and intercept, 0.887 and 0.910 respectively, indicate the null hypothesis cannot be rejected; the inference is that the slope is equal to one and the intercept is equal to zero. However, the t-test analysis that the Rice and Dry Back results are equal in Table 13 show that the null hypothesis can be rejected with the p-value equal to 0.0 which is less than the required 0.05 . This rejection is apparent when examining the means values for the Rice and Dry Back samples, 2.428 and 2.409 respectively.

While the regression analysis states that the slope and intercept are not significantly different from the line of equality, Figure 10 shows a bias between the two samples. If the slope is exactly 1 , then the bias between the two test methods is the intercept of the regression equation. Even though the slope of the regression between the 
Rice and Dry Back methods is not statistically significantly different from 1, the intercept is not an accurate estimator of the bias. The bias is the difference in the overall means of the data of the two test methods. The means of the data sets are 2.428 and 2.409 for the Rice and Dry Back tests respectively. Subtracting the difference, 0.019, from the Rice values adjusts the results for the difference between the methods. Figure 11compares the adjusted Dry Back values to the Rice values. The adjusted Dry Back values agree well with the Rice test results.

If the results of this experiment are applicable to general testing of asphalt then the impact of the bias on volumetric analysis should be considered. If Rice $\mathrm{G}_{\mathrm{mm}}$ results are used without considering the impact of absorptive aggregates then the computed voids in the mix, VTM, will be overestimated by 0.6 to 0.8 percent relative to Dry Back results. For example, if $G_{m b}$ is 2.500 and the Rice $G_{m m}$ is 2.650 the VTM would be 5.7 percent. However, if $\mathrm{G}_{\mathrm{mb}}$ is 2.500 and the Dry Back Gmm is 2.631, the VTM would be 5.0 percent

\subsubsection{Dry Back versus CoreLok}

Figure 12 presents the line of equality graph for the Dry Back versus CoreLok test methods. The p-value for the slope and intercept indicate the null hypothesis cannot be rejected; the inference is that the slope and intercept are not significantly different from one and zero, respectively. The variability in the comparison is much greater than for the comparison between the Rice and CoreLok, due to the variability in the Dry Back results. The bias between the Dry Back and CoreLok is 0.019, indicating the Dry Back results are consistently lower than the CoreLok results.

\subsubsection{Analysis of Conditioning Time}

A main concern for this experiment was the lack of conditioning time of the production samples before testing. For this comparison samples at the design binder content with and without the two hour conditioning time were tested. A graphical result is illustrated in Figure 13. On Figure 13 the bars with hash marks indicate comparisons where the null hypothesis could not be rejected. In five of the nine comparisons for samples with moisture, the null hypothesis could not be rejected. For the dry samples the null hypothesis could not be rejected for two of the nine comparisons. This indicates 
there may be differences in the effect of conditioning depending on the moisture states of the aggregates when the HMAC was blended. A comparison of all the dry samples conditioned for two hours versus the dry samples that were not conditioned determined the null hypothesis could be rejected ( $\mathrm{p}$-value=0.039), inferring the conditioning time affects the $\mathrm{G}_{\mathrm{mm}}$ results. A t-test comparison of all the moist samples conditioned for two hours versus the moist samples that were not conditioned determined the null hypothesis could not be rejected ( $\mathrm{p}$-value $=0.545$ ), inferring the conditioning time does not affect the $\mathrm{G}_{\mathrm{mm}}$ results in moist samples.

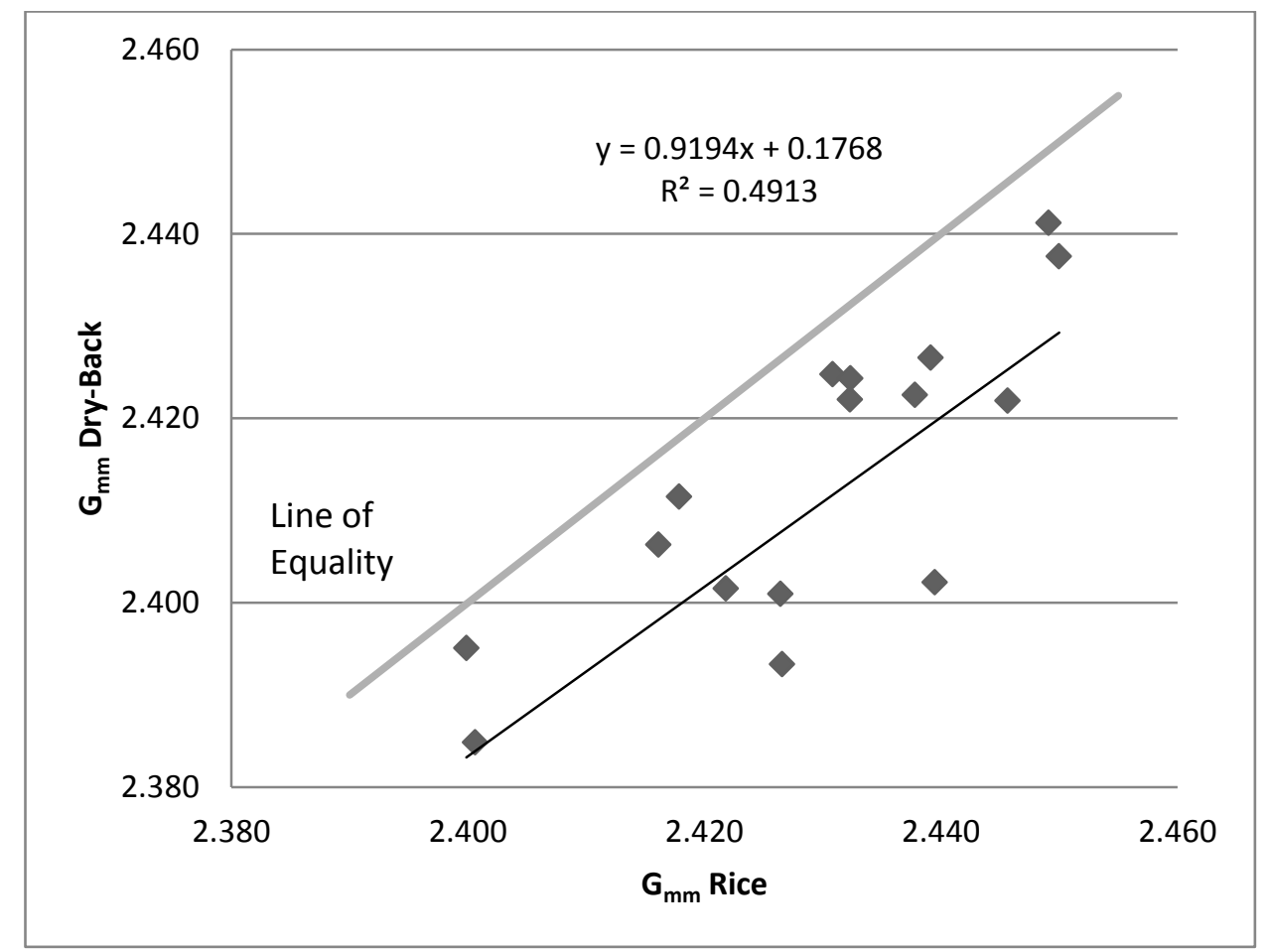

Figure 10 - Line of Equality Chart for Dry Back versus Rice $\mathrm{G}_{\mathrm{mm}}$ Results. 


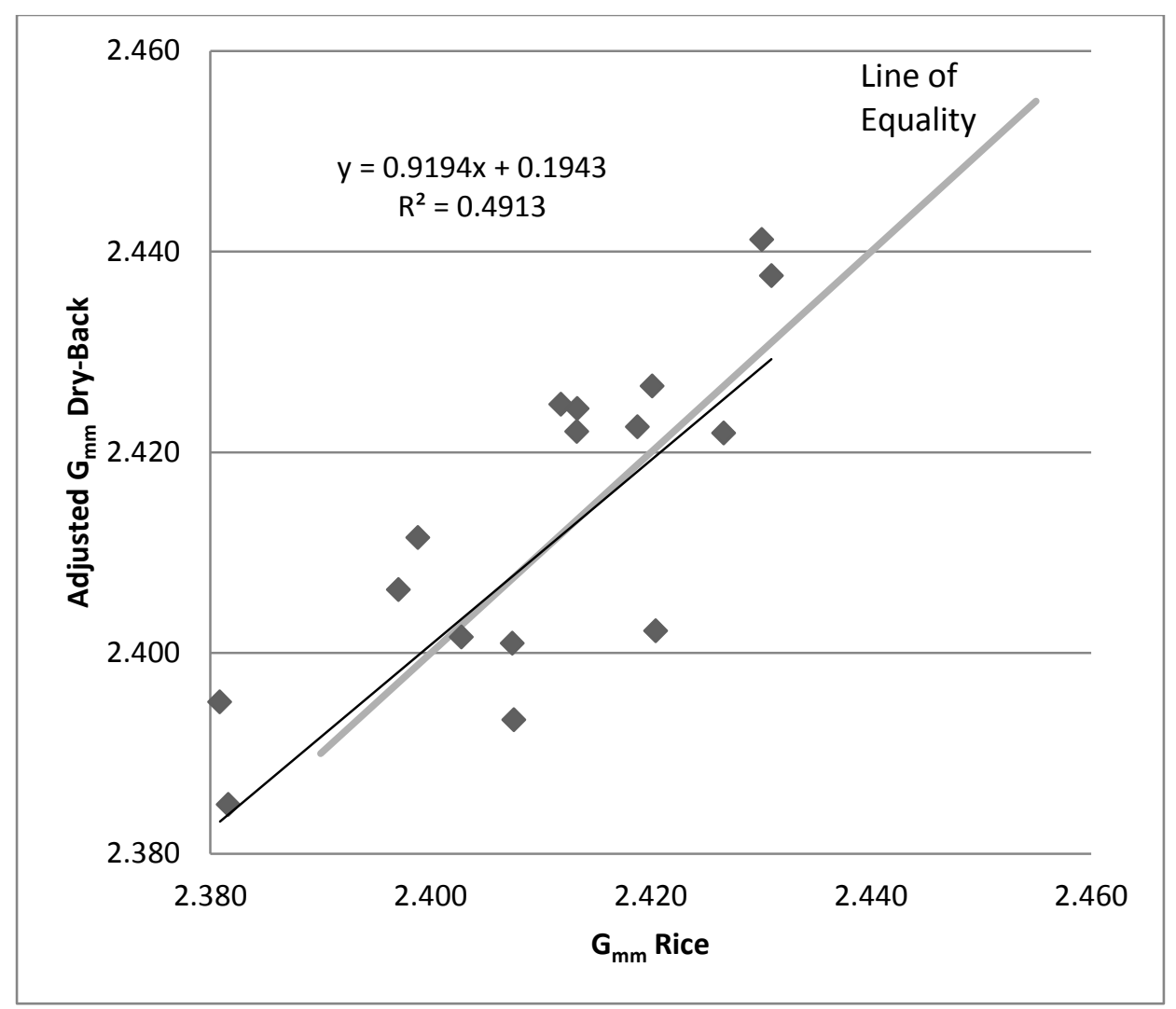

Figure 11 - Adjusted Line of Equality Chart for Dry Back versus Rice $G_{m m}$ Results

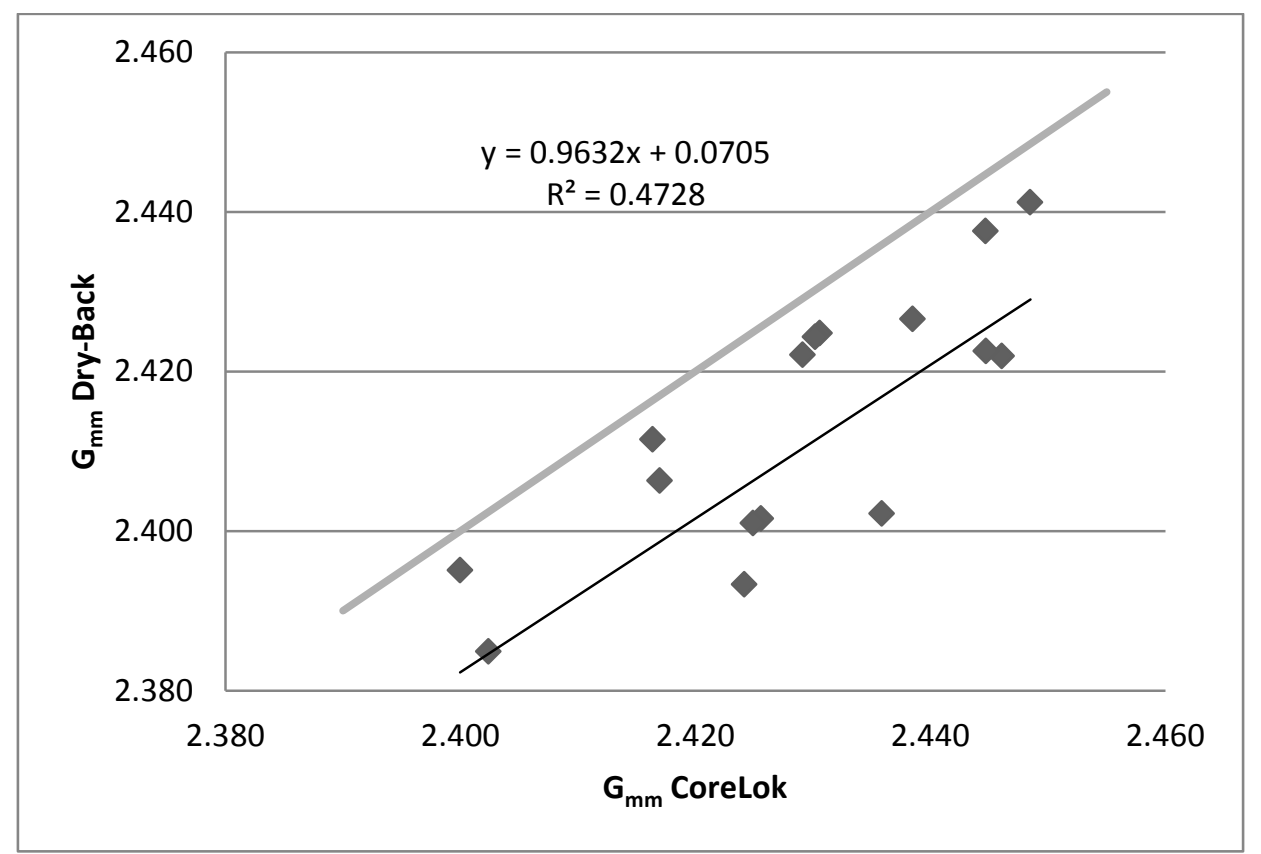

Figure 12 - Line of Equality Chart for Dry Back versus CoreLok $\mathrm{G}_{\mathrm{mm}}$ Results. 


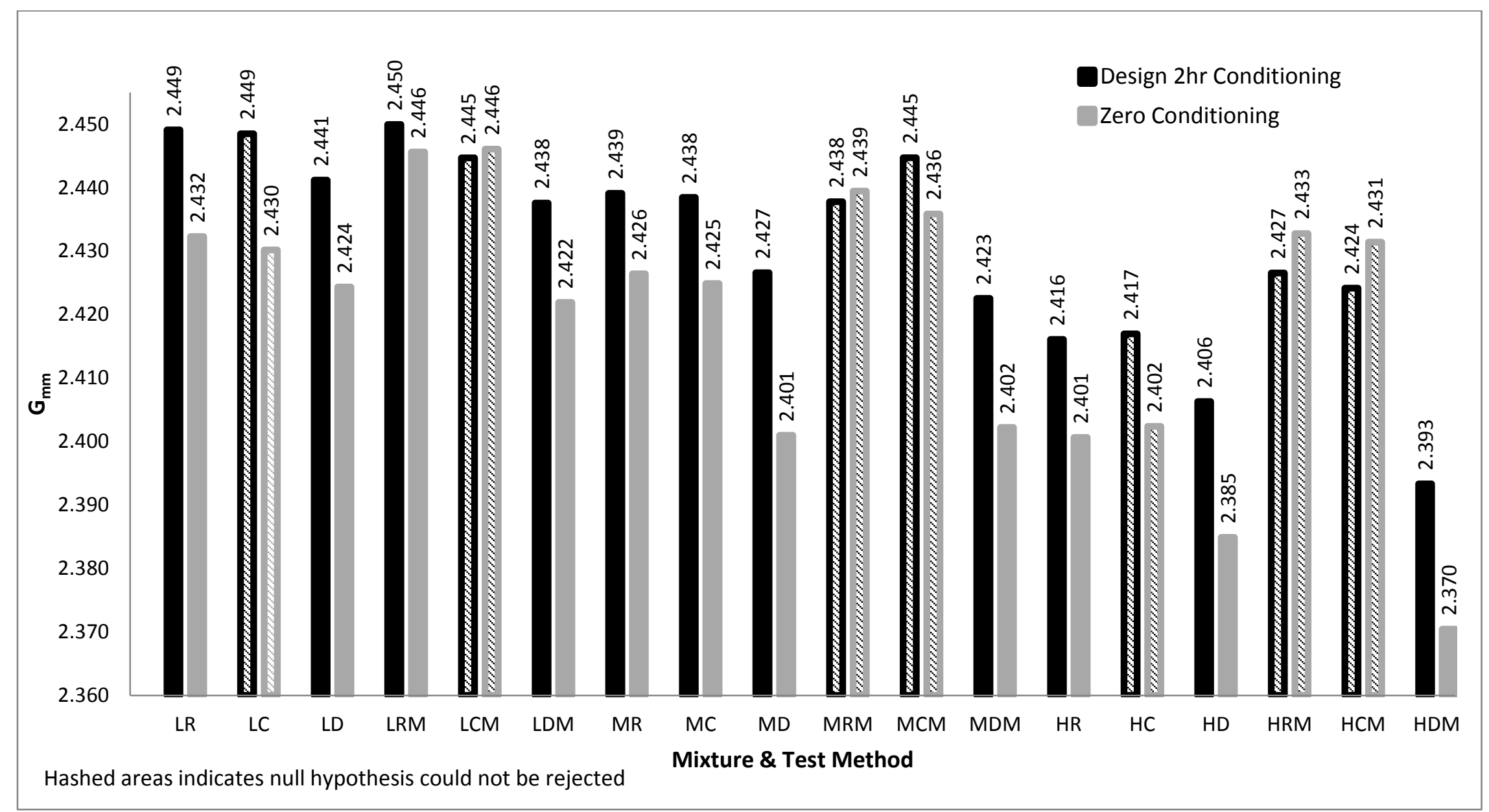

Figure 13 - Conditioning Time Means 


\subsection{3 $\mathrm{G}_{\mathrm{se}}$ Analysis}

For the following comparison, the effective specific gravity of the aggregate, $\mathrm{G}_{\mathrm{se}}$, was computed for each sample. For a given blend of aggregates $\mathrm{G}_{\text {se }}$ should be constant, independent of percent binder, thus $\mathrm{G}_{\mathrm{se}}$ allows a direct comparison of the samples prepared at the design binder content and samples prepared at 0.5 percent above design binder content. $\mathrm{G}_{\mathrm{se}}$ is computed as:

$$
G_{s e}=\frac{P_{s}}{\left(\frac{100}{G_{m m}}-\frac{P_{b}}{G_{b}}\right)}
$$

Where:

$$
\begin{aligned}
& P_{s}=\text { percent weight of the aggregates } \\
& P_{b}=\text { percent weight of the asphalt binder } \\
& G_{s}=\text { specific gravity of the asphalt binder } \\
& G_{s e}=\text { effective specific gravity of aggregates coated in asphalt }
\end{aligned}
$$

\subsubsection{Effects of Slag Content}

The individual $\mathrm{G}_{\mathrm{se}}$ values are presented in Table 15 while Table 16 shows the means and standard deviations of the $\mathrm{G}_{\mathrm{se}}$ values. From the definition of the different types of aggregate specific gravity, $\mathrm{G}_{\mathrm{sb}}<\mathrm{G}_{\mathrm{se}}<\mathrm{G}_{\mathrm{sa}}$. Figure 14 shows that on average this relationship holds. However, the moisture additive samples with high slag contents results of the Rice and CoreLok test methods are suspect of error in that the samples for each method had $\mathrm{G}_{\mathrm{se}}$ values equal to or larger than the $\mathrm{G}_{\mathrm{sa}}$. Samples tested with the Dry Back method did not display this problem.

Figure 14 compares the $\mathrm{G}_{\text {se }}$ values for the three testing methods and the three slag contents. The magnitude of the $\mathrm{G}_{\mathrm{se}}$ constantly decreases going from CoreLok and Rice to the Dry Back. Table 17 is a summary of the t-tests comparing the three test methods for each slag content. At all three slag contents the Rice - CoreLok comparison could not be rejected but both the Rice - Dry Back and the Dry Back - CoreLok comparisons were rejected. This indicates that the Rice and CoreLok produce statistically similar results and the Dry Back method produces statistically different results from the Rice and CoreLok no matter the slag content. 
Table 15 - $\mathrm{G}_{\mathrm{se}}$ Data Sheet

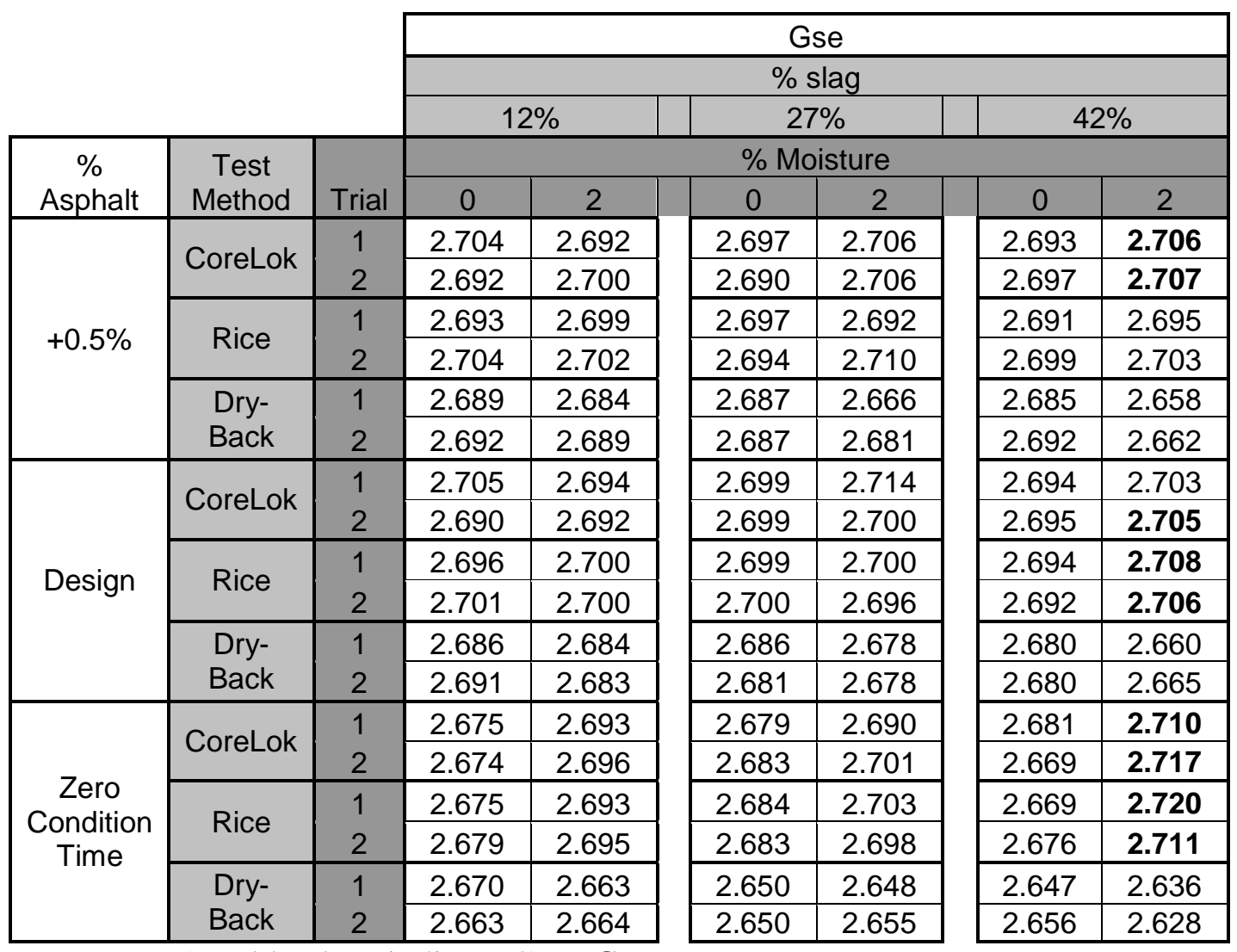

* Bold values indicate $\mathrm{G}_{\mathrm{se}} \geq \mathrm{G}_{\mathrm{sa}}$

Table $16-\mathrm{G}_{\mathrm{se}}$ Means and Standard Deviations

\begin{tabular}{|c|c|c|c|c|c|c|c|c|c|c|c|c|c|}
\hline & \multicolumn{12}{|c|}{ Gse } \\
\hline & & \multicolumn{12}{|c|}{$\%$ slag } \\
\hline & & \multicolumn{4}{|c|}{$12 \%$} & \multicolumn{4}{|c|}{$27 \%$} & \multicolumn{4}{|c|}{$42 \%$} \\
\hline & & \multicolumn{12}{|c|}{$\%$ Moisture } \\
\hline \multirow[b]{2}{*}{$\%$ Asphalt } & \multirow{2}{*}{$\begin{array}{c}\text { Test } \\
\text { Method }\end{array}$} & \multicolumn{2}{|c|}{0} & \multicolumn{2}{|c|}{2} & \multicolumn{2}{|c|}{0} & \multicolumn{2}{|c|}{2} & \multicolumn{2}{|c|}{0} & \multicolumn{2}{|c|}{2} \\
\hline & & Mean & STDEV & Mean & STDEV & Mean & STDEV & Mean & STDEV & Mean & STDEV & Mean & STDEV \\
\hline \multirow{3}{*}{$0.50 \%$} & CoreLok & 2.698 & 0.008 & 2.696 & 0.006 & 2.694 & 0.005 & 2.706 & 0.000 & 2.695 & 0.003 & 2.707 & 0.001 \\
\hline & Rice & 2.699 & 0.008 & 2.701 & 0.002 & 2.696 & 0.002 & 2.701 & 0.013 & 2.695 & 0.006 & 2.699 & 0.006 \\
\hline & Dry-Back & 2.691 & 0.002 & 2.687 & 0.004 & 2.687 & 0.000 & 2.674 & 0.011 & 2.689 & 0.005 & 2.660 & 0.003 \\
\hline \multirow{3}{*}{ Design } & CoreLok & 2.698 & 0.011 & 2.693 & 0.001 & 2.699 & 0.000 & 2.707 & 0.010 & 2.695 & 0.001 & 2.704 & 0.001 \\
\hline & Rice & 2.699 & 0.004 & 2.700 & 0.000 & 2.700 & 0.001 & 2.698 & 0.003 & 2.693 & 0.001 & 2.707 & 0.001 \\
\hline & Dry-Back & 2.689 & 0.004 & 2.684 & 0.001 & 2.684 & 0.004 & 2.678 & 0.000 & 2.680 & 0.000 & 2.663 & 0.004 \\
\hline \multirow{3}{*}{$\begin{array}{c}\text { Zero } \\
\text { Condition } \\
\text { Time }\end{array}$} & CoreLok & 2.675 & 0.001 & 2.695 & 0.002 & 2.681 & 0.003 & 2.696 & 0.008 & 2.675 & 0.008 & 2.714 & 0.005 \\
\hline & Rice & 2.677 & 0.003 & 2.694 & 0.001 & 2.684 & 0.001 & 2.701 & 0.004 & 2.673 & 0.005 & 2.716 & 0.006 \\
\hline & Dry-Back & 2.667 & 0.005 & 2.664 & 0.001 & 2.650 & 0.000 & 2.652 & 0.005 & 2.652 & 0.006 & 2.632 & 0.006 \\
\hline
\end{tabular}




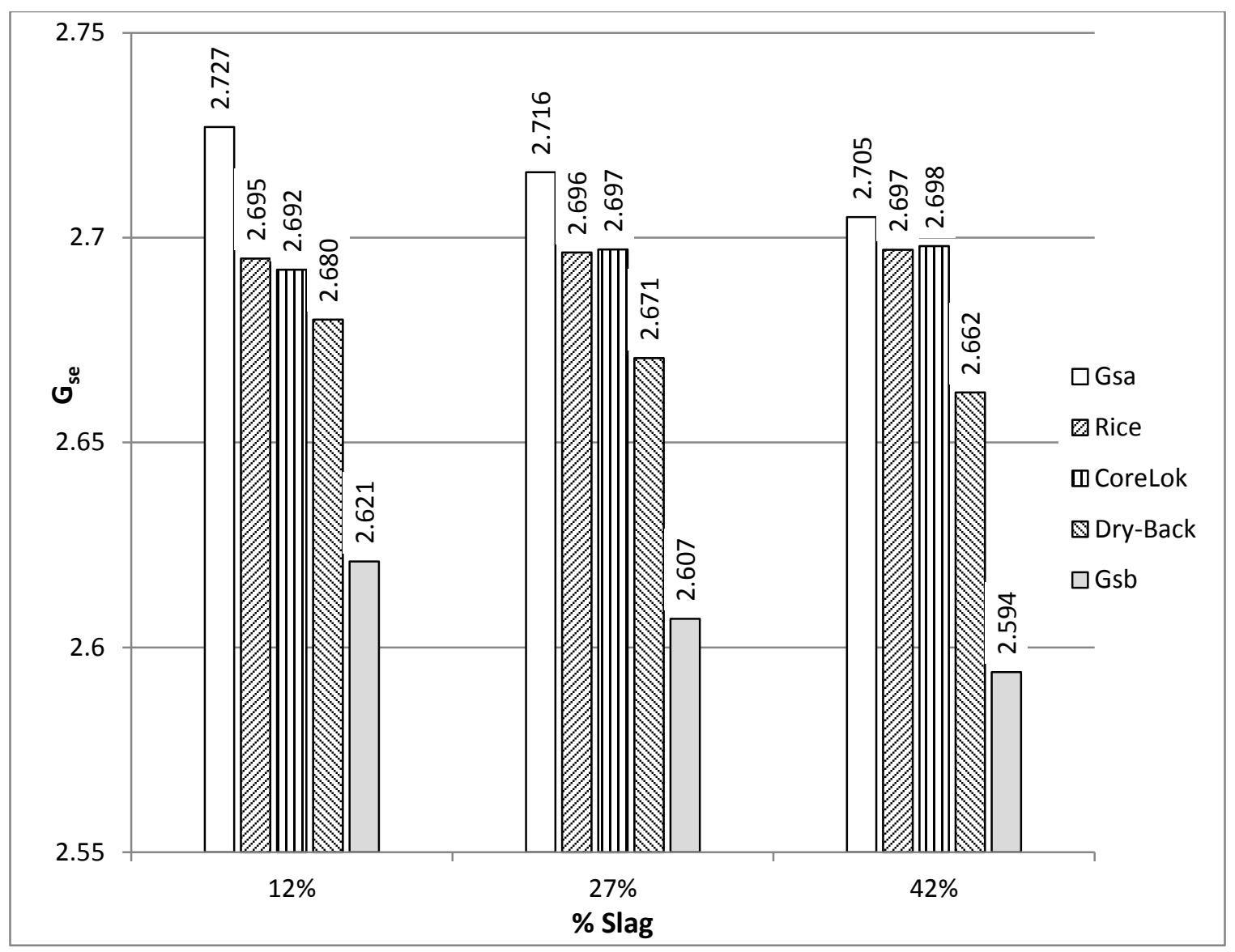

Figure $14-\mathrm{G}_{\mathrm{se}}$ Means

Table 17 - Slag Content Analysis Using $\mathrm{G}_{\mathrm{se}}$

\begin{tabular}{|c|c|c|c|c|c|c|}
\hline \% Slag & Comparison & \multicolumn{2}{|c|}{ Means } & $\begin{array}{c}\text { Degree } \\
\text { of }\end{array}$ & P-value & Decision \\
\hline \multirow{3}{*}{$12 \%$} & Rice-CoreLok & 2.695 & 2.692 & 22 & 0.483 & cannot reject $\mathrm{Hn}$ \\
\hline & Rice-DryBack & 2.695 & 2.680 & 22 & 0.002 & reject $\mathrm{Hn}$ \\
\hline & CoreLok-DryBack & 2.692 & 2.680 & 22 & 0.009 & reject $\mathrm{Hn}$ \\
\hline \multirow{3}{*}{$27 \%$} & Rice-CoreLok & 2.696 & 2.697 & 22 & 0.837 & cannot reject $\mathrm{Hn}$ \\
\hline & Rice-DryBack & 2.696 & 2.671 & 22 & 0.000 & reject $\mathrm{Hn}$ \\
\hline & CoreLok-DryBack & 2.697 & 2.671 & 22 & 0.000 & reject $\mathrm{Hn}$ \\
\hline \multirow{3}{*}{$42 \%$} & Rice-CoreLok & 2.697 & 2.698 & 22 & 0.884 & cannot reject $\mathrm{Hn}$ \\
\hline & Rice-DryBack & 2.697 & 2.662 & 22 & 0.000 & reject $\mathrm{Hn}$ \\
\hline & CoreLok-DryBack & 2.697 & 2.662 & 22 & 0.000 & reject $\mathrm{Hn}$ \\
\hline
\end{tabular}


Figure 15 show a scatter diagram of the $\mathrm{G}_{\text {se }}$ values for each test method and slag content where in each case the values are similar between the CoreLok and Rice and then decrease to the Dry Back. The trend lines from Figure 15 show that the increase in slag content produces a decrease in $\mathrm{G}_{\mathrm{se}}$ for the Dry Back method. This is expected due to the fact that the $G_{s b}$ value for the slag aggregate is lower than the $G_{s b}$ value for the limestone aggregates. Therefore the combined $\mathrm{G}_{\mathrm{sb}}$ for the mix will be lowered with the increase in slag content. However, the Rice and CoreLok do not capture the same trend. These values are relatively the same no matter the slag content. This is possible due to the absorptive nature of the slag and neither test account for account for that. The $\mathrm{R}^{2}$ values from the figure show the high variability of the $\mathrm{G}_{\text {se }}$ values which resulted in not being able to reject the null hypothesis of equal means. Since the variability is high there would be more overlapping of the values which causes a greater probability of equal means.

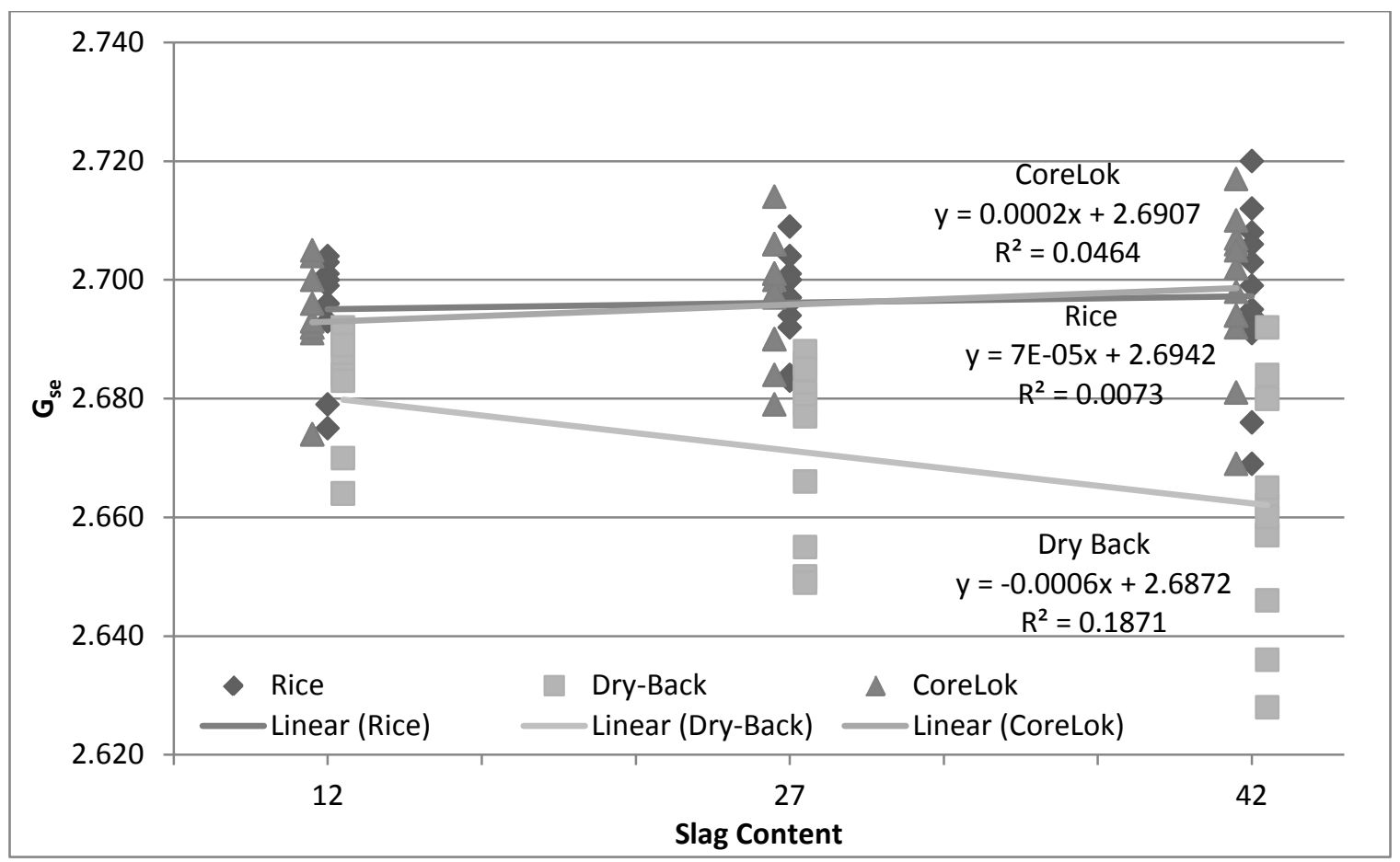

Figure 15 - $\mathrm{G}_{\mathrm{se}}$ Test Data versus Slag Content

\subsubsection{Effect of Additional Binder}

When slag is used in hot mix asphalt there is a chance that since there are large amounts of voids that the aggregates do not get completely coated with asphalt. For the 
experiment a set of samples were prepared with an additional 0.5 percent asphalt in order to overcome this issue and insure adequate coating of the aggregates. Table 18 shows the comparisons for each test method comparing the $G_{\text {se }}$ values at the design binder content and the $\mathrm{G}_{\mathrm{se}}$ values at the design plus 0.5 percent binder content. Table 18 shows that there is little effect on the Gse between the two binder levels for each group of slag percentages. The differences between the results are even lower than the precision statement standard deviation given in AASHTO, with the exception of the two bolded numbers. A statistical analysis was performed and showed that the null hypothesis for equal means could not be rejected for any of the test methods, inferring that there is no statistical difference between the design asphalt content test results and the results from adding additional asphalt. From these comparisons it is apparent that the concept of using additional binder to adequately stabilize the $G_{\text {se }}$ values, and therefore $G_{m m}$ values is not effective, nor desirable.

Table 18 - $\mathrm{G}_{\mathrm{se}}$ Comparison for Additional Binder

\begin{tabular}{|c|c|c|c|c|c|c|}
\hline$\%$ Slag & Test Method & Moisture & Design & $+0.5 \%$ & Difference & Average \\
\hline \multirow{6}{*}{$12 \%$} & \multirow{2}{*}{ CoreLok } & 0 & 2.698 & 2.698 & -0.001 & \multirow{6}{*}{ 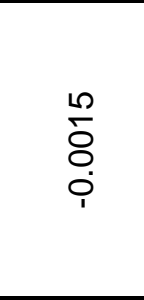 } \\
\hline & & 2 & 2.693 & 2.696 & -0.003 & \\
\hline & \multirow{2}{*}{ Rice } & 0 & 2.699 & 2.699 & 0.000 & \\
\hline & & 2 & 2.700 & 2.701 & 0.000 & \\
\hline & \multirow{2}{*}{ Dry-Back } & 0 & 2.689 & 2.691 & -0.002 & \\
\hline & & 2 & 2.684 & 2.687 & -0.003 & \\
\hline \multirow{6}{*}{$27 \%$} & \multirow{2}{*}{ CoreLok } & 0 & 2.699 & 2.694 & 0.005 & \multirow{6}{*}{$\begin{array}{l}\stackrel{J}{~} \\
\stackrel{8}{\circ} \\
\stackrel{0}{0}\end{array}$} \\
\hline & & 2 & 2.707 & 2.706 & 0.001 & \\
\hline & \multirow{2}{*}{ Rice } & 0 & 2.700 & 2.696 & 0.004 & \\
\hline & & 2 & 2.698 & 2.701 & -0.003 & \\
\hline & \multirow{2}{*}{ Dry-Back } & 0 & 2.684 & 2.687 & -0.003 & \\
\hline & & 2 & 2.678 & 2.674 & 0.005 & \\
\hline \multirow{6}{*}{$42 \%$} & \multirow{2}{*}{ CoreLok } & 0 & 2.695 & 2.695 & -0.001 & \multirow{6}{*}{$\begin{array}{l}\text { 오 } \\
8 \\
\circ \\
\text { ㅇ }\end{array}$} \\
\hline & & 2 & 2.704 & 2.707 & -0.003 & \\
\hline & \multirow{2}{*}{ Rice } & 0 & 2.693 & 2.695 & -0.002 & \\
\hline & & 2 & 2.707 & 2.699 & 0.008 & \\
\hline & \multirow{2}{*}{ Dry-Back } & 0 & 2.680 & 2.689 & -0.009 & \\
\hline & & 2 & 2.663 & 2.660 & 0.002 & \\
\hline & & & & \multicolumn{2}{|c|}{ Average } & -0.0002 \\
\hline
\end{tabular}




\subsubsection{Effect of the Addition of Moisture}

The moisture absorption was addressed in this experiment so a t-test was conducted to see if a significant difference existed between the samples that were mixed normally and those with water induced into the slag portion of the mix.

Figure 16 shows the line of equality between all the dry samples and all the samples with residual moisture.

Figure 16 shows that most of the data are similar, and when examining the regression analysis the slope and intercept are quite close one and zero, respectively. Furthermore the p-values for the slope and intercept are 0.941 and 0.960 respectively, indicating that the null hypothesis cannot be rejected. Also, from the $\mathrm{R}^{2}$ value is 0.65 so there is variability not explained with the regression line. When the data is compared using a t-test with a 95\% confidence level, shown in Table 19, over a third, 10 out of the 27 comparisons can reject the null hypothesis of equal means. Five of the nine zero-conditioned samples can reject the null hypothesis but only five of the 18 two hour conditioned samples can reject the null hypothesis. Also, only two of the nine Dry Back samples rejected the null hypothesis when the Rice and CoreLok both rejected twice that.

Figure 17 shows the line of equality of the mean $\mathrm{G}_{\text {se }}$ values for the zero cured samples comparing the effects of moisture. All of the dry back samples are triangular shaped and are all located at or above the line of equality. The Rice and CoreLok samples are all located below the line of equality. There is insufficient data however to make any good observations, especially due to the high variability in the data.

\subsection{COMPARISON TO PREVIOUS RESEARCH}

The Rajagopal and Crago (2007) study provided results that can be directly compared to the research performed in this report. The data points from Table 2 were entered into Excel to produce a line of equality chart, Figure 18 and a regression analysis shown in Table 20. Table 2 shows the average of the two tests are equal, the analysis conducted in this research show that the tread line is not similar to the predicted intercept and slope values of zero and one respectively. According to the regression analysis the null hypothesis could be rejected for both the slope and the intercept. The R-squared value is 0.5325 , which means there is a much larger variability in the results then the 
results collected in this study. While there is a larger variability in Rajagopal and Crago's results they are explainable due to the high number of technicians working on the same project at once, whereas this study used only one person completed the laboratory work. Ignoring the variability these two studies could have produced similar results for the comparison between the CoreLok and the Rice methods for determining $G_{\mathrm{mm}}$.

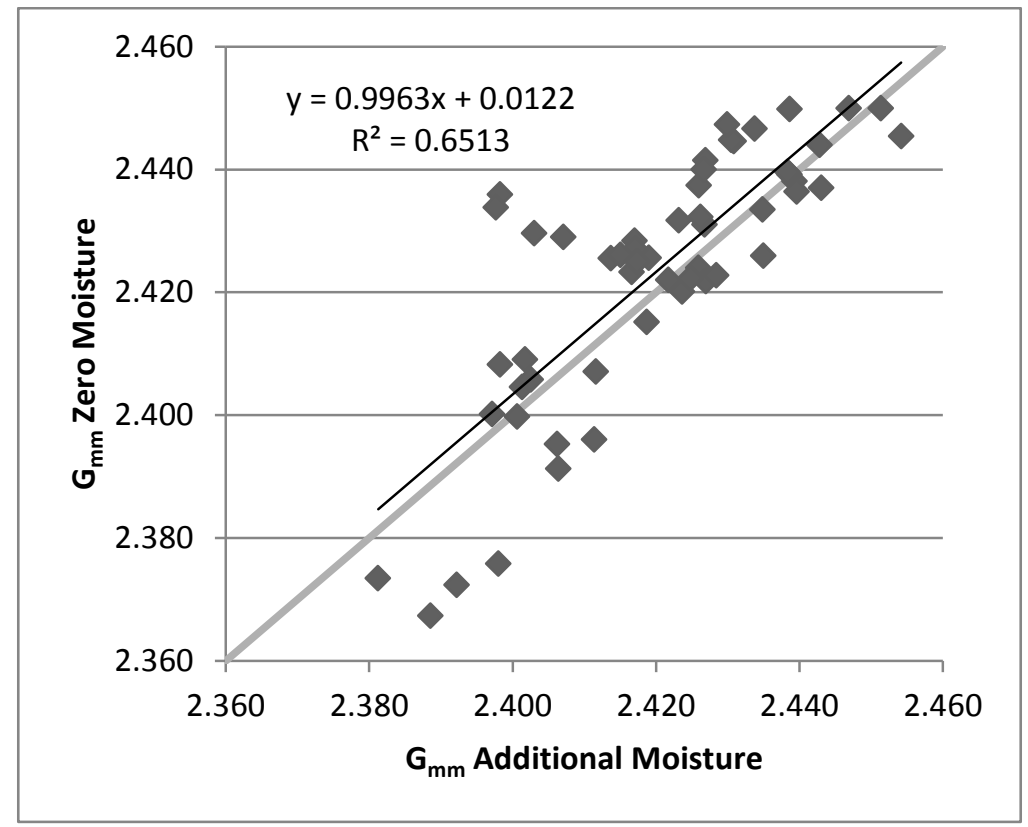

Figure 16 - Line of Equality for Dry vs. Moist sample comparison

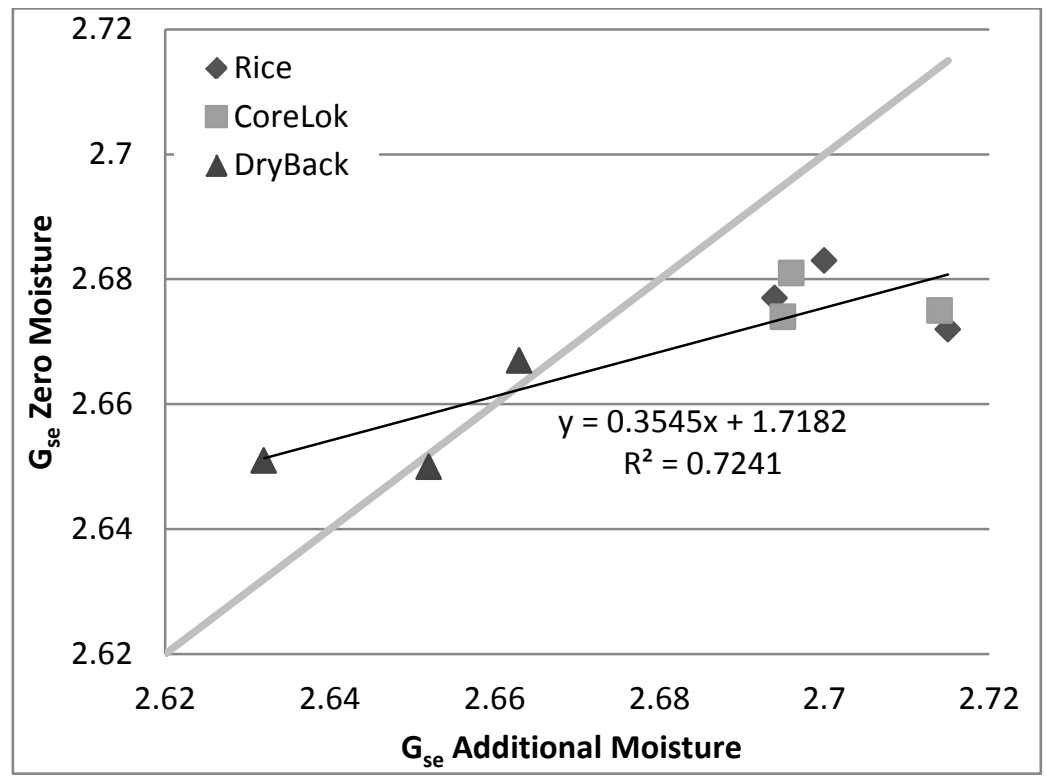

Figure 17 - Moisture Addition Line of Regression for Zero conditioned samples 
Table $19-\mathrm{G}_{\mathrm{mm}}$ Moisture Addition t-test Table

\begin{tabular}{lc|c|cc}
\multicolumn{1}{r}{} & Average & $\begin{array}{c}\text { T-test } \\
\text { (p-values) }\end{array}$ & Average & \\
\cline { 3 - 3 } LPC & 2.431 & 0.76307 & 2.432 & LPCM \\
LPR & 2.431 & 0.81077 & 2.429 & LPRM \\
LPD & 2.425 & 0.34699 & 2.422 & LPDM \\
LDC & 2.449 & 0.73508 & 2.450 & LDCM \\
LDR & 2.449 & 0.57739 & 2.445 & LDRM \\
LDD & 2.441 & 0.20272 & 2.438 & LDDM \\
LZC & 2.432 & 0.01711 & 2.446 & LZCM \\
LZR & 2.430 & 0.00639 & 2.446 & LZRM \\
LZD & 2.424 & 0.45164 & 2.422 & LZDM \\
MPC & 2.418 & 0.61433 & 2.422 & MPCM \\
MPR & 2.416 & 0.07400 & 2.426 & MPRM \\
MPD & 2.411 & 0.21509 & 2.402 & MPDM \\
MDC & 2.439 & 0.46468 & 2.438 & MDCM \\
MDR & 2.438 & 0.34627 & 2.445 & MDRM \\
MDD & 2.427 & 0.15405 & 2.423 & MDDM \\
MZC & 2.426 & 0.02492 & 2.439 & MZCM \\
MZR & 2.425 & 0.13373 & 2.436 & MZRM \\
MZD & 2.401 & 0.65918 & 2.402 & MZDM \\
HPC & 2.400 & 0.50289 & 2.403 & HPCM \\
HPR & 2.400 & 0.04003 & 2.409 & HPRM \\
HPD & 2.395 & 0.02488 & 2.374 & HPDM \\
HDC & 2.416 & 0.01194 & 2.427 & HDCM \\
HDR & 2.417 & 0.01642 & 2.424 & HDRM \\
HDD & 2.406 & 0.02323 & 2.393 & HDDM \\
HZC & 2.401 & 0.01501 & 2.433 & HZCM \\
HZR & 2.402 & 0.03157 & 2.431 & HZRM \\
HZD & 2.385 & 0.09300 & 2.370 & HZDM \\
& & & &
\end{tabular}




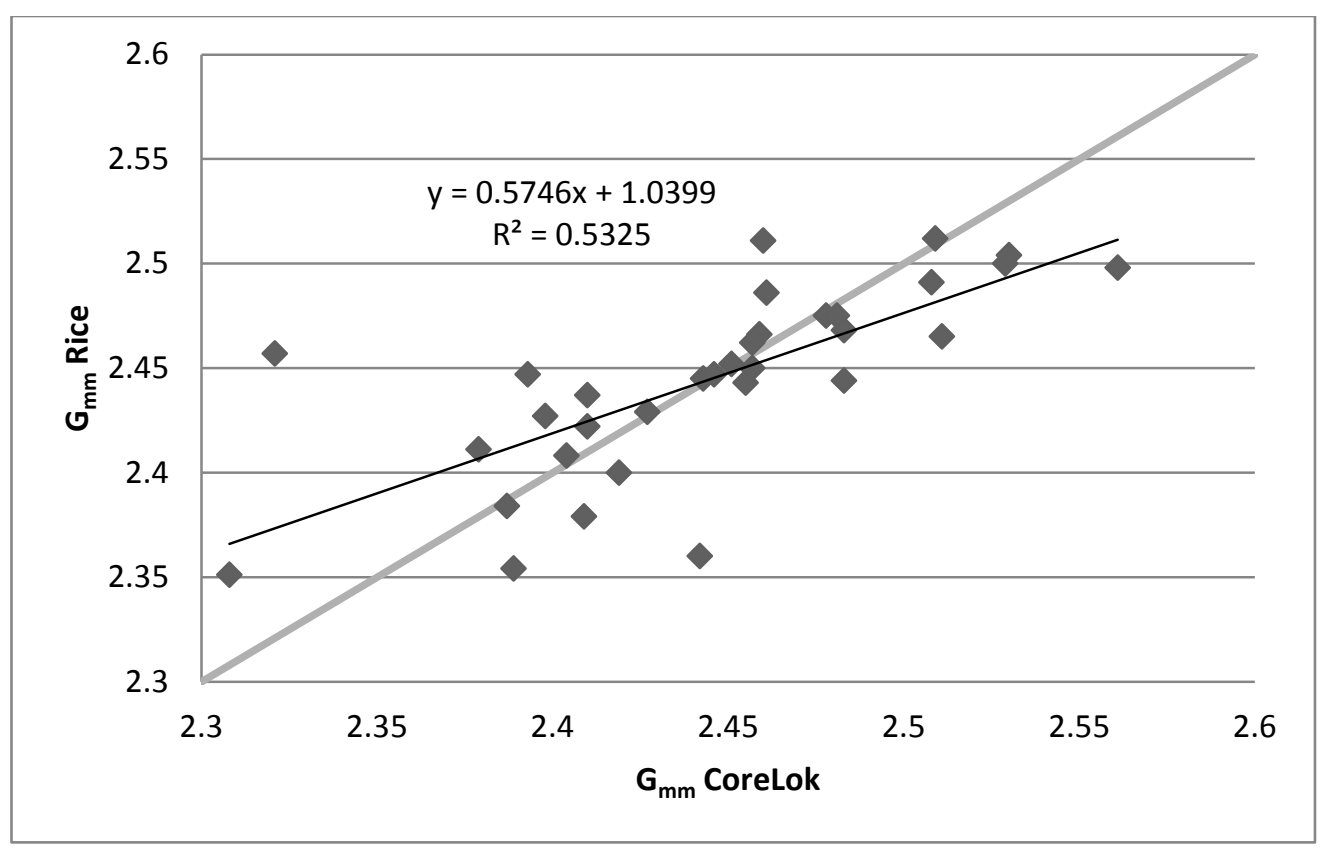

Figure 18 - Line of Equality on Rajagopal and Crago study

Table 20 - Regression analysis on Rajagopal and Crago Study

\begin{tabular}{|c|c|c|c|c|}
\hline \multicolumn{2}{|c|}{ Regression Statistics } & & & \\
\hline Multiple R & 0.729746 & & & \\
\hline R Square & 0.532529 & & & \\
\hline Adjusted R Square & 0.517449 & & & \\
\hline Standard Error & 0.031010 & & & \\
\hline Observations & 33 & & & \\
\hline \multicolumn{5}{|l|}{ ANOVA } \\
\hline & $d f$ & SS & MS & $F$ \\
\hline Regression & 1 & 0.033959 & 0.033959 & 35.314227 \\
\hline Residual & 31 & 0.029811 & 0.000962 & \\
\hline \multirow[t]{2}{*}{ Total } & 32 & 0.063770 & & \\
\hline & Coefficients & Standard Error & t Stat & $P$-value \\
\hline Intercept & 1.03988 & 0.23638 & 4.39910 & 0.00012 \\
\hline X Variable 1 & 0.57457 & 0.09669 & 5.94258 & 0.00000 \\
\hline tails & 2 & & Decision & \\
\hline $\mathrm{t}$ for $\mathrm{Hn}=1$ & -4.400 & & Intercept & reject $\mathrm{Hn}$ \\
\hline $\mathrm{p}$-value for $\mathrm{Hn}=1$ & 0.000 & & Slope & reject $\mathrm{Hn}$ \\
\hline
\end{tabular}




\section{CHAPTER 5 CONCLUSIONS AND RECOMMENDATIONS}

\subsection{CONCLUSIONS}

The first objective of this study was to determine a possible cause for the difference in theoretical maximum specific gravity $\left(\mathrm{G}_{\mathrm{mm}}\right)$ results between a laboratory created sample and a sample pulled from the production line in the plant. Possible causes thought to affect this were the residual moisture that is held in the slag after it passes through the drying drum and the lack of any conditioning time as the samples are pulled from the plant for testing. Both of these were tested and analyzed.

The analysis compared multiple mixture designs, asphalt contents, and test methods. The addition of water prior to the mixing procedure revealed minimal influence on the end values for theoretical maximum specific gravity, only affecting one-third of the values. It is important to point out that about seventy percent of those affected were found to be in the high slag content mixture. There was also an increase with the variability of the results when moisture was induced in the samples. Even though the moisture had an effect on the test data, overall it was in the wrong direction. The moist samples had $G_{m m}$ values were higher than those samples with dry aggregates.

The second theory involving the lack of a conditioning time for plant produced samples proved to be probable. With 75 percent of the samples showing significant differences between the standard two hours and the simulated plant produced sample with no conditioning. There were only two of nine samples without residual moisture that the null hypothesis could not be rejected, inferring that there is a significant difference between conditioning times.

The second objective was to determine if the Dry Back procedure was necessary. According to the $G_{\text {se }}$ results the Dry Back samples were the only samples to account for the effects of the additional slag. Figure 15 showed that the Dry Back values were the only ones to decrease as the slag content increase. This indicates the standard method is not capturing the effect of absorption; hence there is no basis for discontinuing the use of the Dry Back method at this time. 
The final objective was to determine if the CoreLok could replace the Rice test (AASHTO T-209). The $\mathrm{G}_{\mathrm{mm}}$ analysis for the test methods revealed there was a strong correlation between all the testing methods. The CoreLok showed no statistical difference from the Rice method results. While there is a strong correlation between the Rice and CoreLok there was bias when comparing the Dry Back to the Rice and CoreLok. The CoreLok and Rice test methods always overestimate the Dry Back method results. The bias between the Rice and Dry Back and between the CoreLok and Dry Back were equal to 0.019; this is interesting because InstroTek claims that the CoreLok eliminates the need for the Dry Back procedure. Due to these and the results conducted by the University of Cincinnati, it appears that the CoreLok can be used in lieu of T-209 if there are no absorptive aggregates being used in the mix design. However, if there are absorptive aggregates then the Dry Back will need to be used to account for the absorption.

Table 21 is a list of statistical analyses used when comparing the results. Other $\mathrm{G}_{\mathrm{se}}$ analysis was conducted to be able to compare results between design binder content and samples with the additional 0.5 percent binder more accurately then $G_{m m}$ can. This is because $G_{s e}$ is not dependent on the asphalt content like $G_{m m}$ is. These analyses revealed that adding additional binder to aid in sealing the stones better had little influence on the end results. Since $\mathrm{G}_{\mathrm{se}}$ had been calculated, $\mathrm{F}$, the absorption factor was calculated. $\mathrm{F}$ captures the difference in the amount of asphalt absorbed in the aggregate compared to the amount of water absorbed. These values while not included in this report since the analysis would show the same results the $G_{s e}$ had. However, the data did show that there was a significant difference from the AASHTO assumed value of 0.8 , which may have to do with the high void content in slag aggregates.

\subsection{RECOMMENDATIONS FOR FURTHER STUDY}

This research was limited to following the certain test procedure associated with the three test methods used. Knowing this, further research could be conducted to analyze the potential need for a Dry Back procedure for the CoreLok vacuum sealing device. The researched mixture designs for this thesis were limited to only slag No.8, without the use of slag sands. Further investigations should be conducted to see the 
Table 21 - Summary of Analytical Tests

\begin{tabular}{|c|c|c|}
\hline $\begin{array}{l}\text { Statistical } \\
\text { Test }\end{array}$ & Data Tested & Results \\
\hline T-test & $\begin{array}{l}\mathrm{G}_{\mathrm{mm}} \text { data for each } \\
\text { Test Method }\end{array}$ & $\begin{array}{l}\text { Null hypothesis could not be rejected between Rice } \\
\text { and CoreLok. } \\
\text { Null hypothesis was rejected between Dry Back and } \\
\text { Rice and CoreLok. }\end{array}$ \\
\hline $\begin{array}{l}\text { Linear } \\
\text { Regression }\end{array}$ & $\begin{array}{l}\mathrm{G}_{\mathrm{mm}} \text { data for each test } \\
\text { method }\end{array}$ & $\begin{array}{l}\text { Showed the Rice and CoreLok procedures produced } \\
\text { very similar results. } \\
\text { Showed that the Dry Back data was correlated to } \\
\text { the Rice and CoreLok but offset by } 0.019 \text {. }\end{array}$ \\
\hline T-test & $\begin{array}{l}\mathrm{G}_{\mathrm{mm}} \text { data with design } \\
\text { binder contents }\end{array}$ & $\begin{array}{l}\text { Null hypothesis could be rejected with dry samples. } \\
\text { Null hypothesis could not be rejected with moist } \\
\text { samples due to high variability. }\end{array}$ \\
\hline T-test & $\begin{array}{l}\mathrm{G}_{\mathrm{se}} \text { data separated by } \\
\text { slag contents }\end{array}$ & $\begin{array}{l}\text { Null hypothesis could not be rejected between Rice } \\
\text { and CoreLok. } \\
\text { Null hypothesis was rejected between Dry Back and } \\
\text { Rice and CoreLok. }\end{array}$ \\
\hline & $\begin{array}{l}\mathrm{G}_{\mathrm{se}} \text { data separated by } \\
\text { slag contents }\end{array}$ & $\begin{array}{l}\text { As slag content increased } G_{s e} \text { became closer to the } \\
G_{\text {sa }} \text { upper limit especially when studying the } \\
\text { samples with residual moisture. }\end{array}$ \\
\hline $\begin{array}{l}\text { Mean } \\
\text { differences }\end{array}$ & $\begin{array}{l}\mathrm{G}_{\text {se }} \text { data between } \\
\text { standard mix design } \\
\text { and mix design with } \\
\text { additional } 0.5 \text { percent } \\
\text { binder }\end{array}$ & $\begin{array}{l}\text { Reveled little difference between the samples, less } \\
\text { variability then the precision statement in } \\
\text { AASHTO's T209 permits, with the exception of } \\
\text { four samples. }\end{array}$ \\
\hline T-test & $\begin{array}{l}\mathrm{G}_{\mathrm{se}} \text { data between dry } \\
\text { and moist mixtures }\end{array}$ & $\begin{array}{l}\text { The null hypothesis could be rejected in } 10 \text { of the } \\
27 \text { comparisons with } 70 \text { percent being high slag } \\
\text { mixtures. }\end{array}$ \\
\hline $\begin{array}{l}\text { Linear } \\
\text { Regression }\end{array}$ & $\begin{array}{l}\mathrm{G}_{\mathrm{se}} \text { data between dry } \\
\text { and moist mixtures }\end{array}$ & $\begin{array}{l}\text { Overall the data was correlated over the line of } \\
\text { equality but had a mediocre r-squared value due to } \\
\text { the variability in the moist samples. }\end{array}$ \\
\hline
\end{tabular}


possible influence that different size aggregates have on the change in testing results. Also, the addition of slag sand instead of limestone sand could have influence in the $\mathrm{G}_{\mathrm{mm}}$ results. The mixtures were also limited to a maximum of 42 percent slag to resemble a high amount of slag present in mixtures. From discussions with West Virginia Paving, the highest amount of course slag they had ever used in a mixture was 50 percent. It is possible that in extreme cases, where slag is the abundant material, the moisture addition could become even more apparent.

As conditioning time appears to be a large contributor to the change in theoretical maximum specific gravity results, further investigations should be done to see the effects at various times. The research should explore the possibility of adding additional short conditioning times (15 to 30 minutes) to plant samples to best account for the absorption of certain aggregates. Also, possible research could be conducted to analyze the absorption factor, F, for absorbent materials such as slag, that way mix designs can better resemble the material used. 


\section{REFERENCES}

FHWA, 1997, User Guidelines for Waste and Byproduct Materials in Pavement Construction.Blast Furnace Slag: Material Description. Publication Number:FHWA-RD-97-148. http://www.fhwa.dot.gov/publications/ research/infrastructure/pavements/97148/008.cfm 1997. 8/16/11.

FHWA, 2010, A Review of Aggregate and Asphalt Mixture Specific Gravity and Their Impacts on Asphalt Mix Design Properties and Mix Acceptance, Tech Brief FHWA-HIF-11-033.

InstroTek Inc., 2011, CoreLok Operator’s Guide. V.23 http://www.instrotek.com/pdfs/CoreLok_Manual.pdf

InstroTek Inc., 2011, CoreLok Aggregate Gravity \& Absorption Test http://www.instrotek.com/pdfs/Corelok\%20Aggplus.pdf

Rajagopal, A., and Crago, D., 2007, A comparative evaluation of the corelok device in determining reliable bulk specific gravity and maximum specific gravity test results. FHWA/OH-2007/07, Ohio Department of Transportation, Columbus, $\mathrm{OH}$.

Sholar, G.A., G. Page,J. Musselman, P. Upshaw, and H. Mosley, 2003,Investigation of the CoreLok for Maximum, Aggregate and Bulk Specific Gravity Test. Research Report FL/DOT/SMO/03-462.

Van Oss, H., 2007 Minerals Yearbook,Slag-Iron and Steel,US Geological Survey. 


\section{APPENDIX - DATA SHEETS}

Table 22 - LD DATA

LD

43 LS - 12 Slag - 45 Sand

\begin{tabular}{|c|c|}
\hline \multicolumn{2}{|c|}{ Rice } \\
\hline Dry & 1508 \\
\hline Submerged & 2146.7 \\
\hline $\mathrm{Gmm}$ & 2.447 \\
\hline Dry & 1516.4 \\
\hline Submerged & 2152.8 \\
\hline $\mathrm{Gmm}$ & 2.451 \\
\hline
\end{tabular}

Dry Back
\begin{tabular}{|l|r|r|}
\hline Dry & \multicolumn{2}{|c|}{1508} \\
\hline Submerged & \multicolumn{2}{|c|}{2146.7} \\
\hline Drying Rack & \multicolumn{2}{|c|}{7334.6} \\
\hline DB 1 & 8845.9 & 1511.3 \\
\hline DB 2 & 8845 & 1510.4 \\
\hline DB 3 & 8844.5 & 1509.9 \\
\hline DB 4 & & \\
\hline DB 5 & \multicolumn{2}{|c|}{} \\
\hline Gmm & \multicolumn{2}{|c|}{2.439} \\
\hline
\end{tabular}

CoreLok
\begin{tabular}{|l|r|}
\hline Bags & 75 \\
\hline Dry & 1509.2 \\
\hline Sub + Bags & 886.2 \\
\hline Gmm & 2.454 \\
\hline \multicolumn{2}{|l|}{} \\
\hline Bags & 74.4 \\
\hline Dry & 1514.8 \\
\hline Sub + Bags & 886.7 \\
\hline Gmm & 2.443 \\
\hline
\end{tabular}

\begin{tabular}{|l|r|r|}
\hline Dry & \multicolumn{2}{|c|}{1516.4} \\
\hline Submerged & \multicolumn{2}{|c|}{2152.8} \\
\hline Drying Rack & \multicolumn{2}{|c|}{6312.2} \\
\hline DB 1 & 7835.3 & 1523.1 \\
\hline DB 2 & 7832.2 & 1520 \\
\hline DB 3 & 7831.2 & 1519 \\
\hline DB 4 & 7830.7 & 1518.5 \\
\hline DB 5 & & \\
\hline Gmm & \multicolumn{2}{|c|}{2.443} \\
\hline \multicolumn{3}{|c|}{ Dry Back }
\end{tabular}

Rice

$\begin{array}{cc}\text { Averages } & 2.449 \\ \text { Stddev } & 0.0032\end{array}$

Averages

2.441

Stddev

0.0026

CoreLok

Averages $\quad 2.449$

Stddev $\quad 0.0081$ 
Table 23 - MD DATA

$$
\begin{gathered}
\text { MD } \\
28 \text { LS - } 27 \text { Slag - } 45 \text { Sand }
\end{gathered}
$$

Rice
\begin{tabular}{|l|r|}
\hline Dry & 1505.6 \\
\hline Submerged & 2143.2 \\
\hline Gmm & 2.439 \\
\hline \multicolumn{2}{|l|}{} \\
\hline Dry & 1515.5 \\
\hline Submerged & 2149.3 \\
\hline Gmm & 2.440 \\
\hline
\end{tabular}

\begin{tabular}{|l|r|l|}
\multicolumn{3}{|c|}{ Dry Back } \\
\hline Dry & \multicolumn{2}{|c|}{1505.6} \\
\hline Submerged & \multicolumn{2}{|c|}{2143.2} \\
\hline Drying Rack & \multicolumn{2}{|c|}{7334.5} \\
\hline DB 1 & 8849.4 & 1514.9 \\
\hline DB 2 & 8845.6 & 1511.1 \\
\hline DB 3 & 8844 & 1509.5 \\
\hline DB 4 & 8843 & 1508.5 \\
\hline DB 5 & 8842.7 & 1508.2 \\
\hline Gmm & \multicolumn{2}{|c|}{2.428} \\
\hline
\end{tabular}

\begin{tabular}{|l|r|}
\hline Bags & 74.3 \\
\hline Dry & 1518.1 \\
\hline Sub + Bags & 887.6 \\
\hline Gmm & 2.439 \\
\hline \multicolumn{2}{|l|}{} \\
\hline Bags & 74.2 \\
\hline Dry & 1497.2 \\
\hline Sub + Bags & 875.2 \\
\hline Gmm & 2.438 \\
\hline
\end{tabular}

\begin{tabular}{|l|c|l|}
\hline Dry & \multicolumn{2}{|c|}{1515.5} \\
\hline Submerged & \multicolumn{2}{|c|}{2149.3} \\
\hline Drying Rack & \multicolumn{2}{|c|}{6313} \\
\hline DB 1 & 7842.2 & 1529.2 \\
\hline DB 2 & 7835.4 & 1522.4 \\
\hline DB 3 & 7833.8 & 1520.8 \\
\hline DB 4 & 7832.8 & 1519.8 \\
\hline DB 5 & 7832.3 & 1519.3 \\
\hline Gmm & \multicolumn{2}{|c|}{2.425} \\
\hline
\end{tabular}

Rice

Dry Back

\section{CoreLok}

Averages $\quad 2.439$

Averages

2.427

Stddev $\quad 0.0007$

Stddev

0.0025

Averages $\quad 2.438$

Stddev $\quad 0.0002$ 
Table 24 - HD DATA

HD

13 LS - 42 Slag - 45 Sand

Rice
\begin{tabular}{|l|r|}
\hline Dry & 1511.2 \\
\hline Submerged & 2141 \\
\hline Gmm & 2.417 \\
\hline \multicolumn{2}{|l|}{} \\
\hline Dry & 1514 \\
\hline Submerged & 2142.1 \\
\hline Gmm & 2.415 \\
\hline
\end{tabular}

\begin{tabular}{|l|c|c|}
\multicolumn{2}{c}{ Dry Back } \\
\hline Dry & \multicolumn{2}{|c|}{1511.2} \\
\hline Submerged & \multicolumn{2}{|c|}{2141} \\
\hline Drying Rack & \multicolumn{2}{|c|}{7334.5} \\
\hline DB 1 & 8849.5 & 1515 \\
\hline DB 2 & 8848.5 & 1514 \\
\hline DB 3 & & \\
\hline DB 4 & & \\
\hline DB 5 & \multicolumn{2}{|c|}{} \\
\hline Gmm & \multicolumn{2}{|c|}{2.406} \\
\hline
\end{tabular}

\begin{tabular}{|l|r|}
\hline Bags & 74.6 \\
\hline Dry & 1492.7 \\
\hline Sub + Bags & 867 \\
\hline Gmm & 2.417 \\
\hline \multicolumn{2}{|l|}{} \\
\hline Bags & 74.2 \\
\hline Dry & 1531.7 \\
\hline Sub + Bags & 890.1 \\
\hline Gmm & 2.417 \\
\hline
\end{tabular}

\begin{tabular}{|l|r|r|}
\hline Dry & \multicolumn{2}{|c|}{1514} \\
\hline Submerged & \multicolumn{2}{|c|}{2142.1} \\
\hline Drying Rack & \multicolumn{2}{|c|}{6312.2} \\
\hline DB 1 & 7832 & 1519.8 \\
\hline DB 2 & 7829 & 1516.8 \\
\hline DB 3 & 7828.5 & 1516.3 \\
\hline DB 4 & & -6312 \\
\hline DB 5 & \multicolumn{2}{|c|}{} \\
\hline Gmm & \multicolumn{2}{|c|}{2.406} \\
\hline
\end{tabular}

Rice

Dry Back

\section{CoreLok}

Averages

2.416

Averages

2.406

0.0001
Averages $\quad 2.417$

Stddev $\quad 0.0005$ 
Table 25 - LDM DATA

LDM
43 LS - 12 Slag - 45 Sand + 2\%MC

Rice

\begin{tabular}{l|r|}
\hline Dry & 1509.2 \\
\hline Submerged & 2148.2 \\
\hline Gmm & 2.450 \\
\hline \multicolumn{2}{|l}{} \\
\hline Dry & 1514.8 \\
\hline Submerged & 2151.5 \\
\hline Gmm & 2.450 \\
\hline
\end{tabular}

Rice

Averages

Stddev
2.450

0.0000
Dry Back

\begin{tabular}{|l|c|c|}
\hline Dry & \multicolumn{2}{|c|}{1509.2} \\
\hline Submerged & \multicolumn{2}{|c|}{2147.2} \\
\hline Drying Rack & \multicolumn{2}{|c|}{6312.5} \\
\hline DB 1 & 7823.7 & 1511.2 \\
\hline DB 2 & 7825.6 & 1513.1 \\
\hline DB 3 & 7824.9 & 1512.4 \\
\hline DB 4 & & \\
\hline DB 5 & \multicolumn{2}{|c|}{} \\
\hline Gmm & \multicolumn{2}{|c|}{2.438} \\
\hline
\end{tabular}

\begin{tabular}{|l|c|l|}
\hline Dry & \multicolumn{2}{|c|}{1514.87} \\
\hline Submerged & \multicolumn{2}{|c|}{2151.5} \\
\hline Drying Rack & \multicolumn{2}{|c|}{7334.5} \\
\hline DB 1 & 8860.8 & 1526.3 \\
\hline DB 2 & 8856.4 & 1521.9 \\
\hline DB 3 & 8854.1 & 1519.6 \\
\hline DB 4 & 8853.1 & 1518.6 \\
\hline DB 5 & 8852.6 & 1518.1 \\
\hline Gmm & \multicolumn{2}{|c|}{2.437} \\
\hline
\end{tabular}

Dry Back

Averages $\quad 2.438$

Stddev
CoreLok

\begin{tabular}{|l|r|}
\hline Bags & 74.1 \\
\hline Dry & 1456.6 \\
\hline Sub + Bags & 853 \\
\hline Gmm & 2.445 \\
\hline \multicolumn{2}{|l}{} \\
\hline Bags & 74.8 \\
\hline Dry & 1571.4 \\
\hline Sub + Bags & 920.4 \\
\hline Gmm & 2.444 \\
\hline
\end{tabular}

\section{CoreLok}

Averages $\quad 2.445$

Stddev $\quad 0.0010$ 
Table 26 - MDM DATA

MDM
28 LS - 27 Slag - 45 Sand $+2 \%$ MC

Rice

\begin{tabular}{|l|r|}
\hline Dry & 1510.1 \\
\hline Submerged & 2146 \\
\hline Gmm & 2.439 \\
\hline \multicolumn{2}{|l}{} \\
\hline Dry & 1509.6 \\
\hline Submerged & 2145 \\
\hline Gmm & 2.436 \\
\hline
\end{tabular}

Rice

Averages $\quad 2.438$

Stddev $\quad 0.0020$
Dry Back

\begin{tabular}{|l|r|l|}
\hline Dry & \multicolumn{2}{|c|}{1510.1} \\
\hline Submerged & \multicolumn{2}{|c|}{2146} \\
\hline Drying Rack & \multicolumn{2}{|c|}{7334.5} \\
\hline DB 1 & 8858.2 & 1523.7 \\
\hline DB 2 & 8851.7 & 1517.2 \\
\hline DB 3 & 8850 & 1515.5 \\
\hline DB 4 & 8849.2 & 1514.7 \\
\hline DB 5 & 8848.8 & 1514.3 \\
\hline Gmm & \multicolumn{2}{|c|}{2.423} \\
\hline
\end{tabular}

\begin{tabular}{|l|c|c|}
\hline Dry & \multicolumn{2}{|c|}{1509.6} \\
\hline Submerged & \multicolumn{2}{|c|}{2145} \\
\hline Drying Rack & 6312.2 \\
\hline DB 1 & 7829.5 & 1517.3 \\
\hline DB 2 & 7826.6 & 1514.4 \\
\hline DB 3 & 7825.7 & 1513.5 \\
\hline DB 4 & 7825.4 & 1513.2 \\
\hline DB 5 & \multicolumn{2}{|c|}{} \\
\hline Gmm & \multicolumn{2}{|c|}{2.422} \\
\hline
\end{tabular}

Dry Back

Averages $\quad 2.423$

Stddev $\quad 0.0003$
CoreLok

\begin{tabular}{l|r|}
\hline Bags & 74.5 \\
\hline Dry & 1526 \\
\hline Sub + Bags & 895.1 \\
\hline Gmm & 2.450 \\
\hline \multicolumn{2}{|l}{} \\
\hline Bags & 74.8 \\
\hline Dry & 1495.4 \\
\hline Sub + Bags & 874.4 \\
\hline Gmm & 2.440 \\
\hline
\end{tabular}

\section{CoreLok}

Averages $\quad 2.445$

Stddev $\quad 0.0072$ 
Table 27 - HDM DATA

\section{HDM \\ 13 LS - 42 Slag - 45 Sand $+2 \%$ MC}

\begin{tabular}{|c|c|}
\hline \multicolumn{2}{|c|}{ Rice } \\
\hline Dry & 1510.6 \\
\hline Submerged & 2143.2 \\
\hline $\mathrm{Gmm}$ & 2.427 \\
\hline Dry & 1512.9 \\
\hline Submerged & 2144.3 \\
\hline $\mathrm{Gmm}$ & 2.426 \\
\hline
\end{tabular}

\begin{tabular}{|l|r|l|}
\multicolumn{2}{|c|}{ Dry Back } \\
\hline Dry & \multicolumn{2}{|c|}{1510.6} \\
\hline Submerged & 2143.2 \\
\hline Drying Rack & \multicolumn{2}{|c|}{6312.5} \\
\hline DB 1 & 7837 & 1524.5 \\
\hline DB 2 & 7834.4 & 1521.9 \\
\hline DB 3 & 7833.3 & 1520.8 \\
\hline DB 4 & 7832.6 & 1520.1 \\
\hline DB 5 & 7832.4 & 1519.9 \\
\hline Gmm & \multicolumn{2}{|c|}{2.391} \\
\hline
\end{tabular}

\begin{tabular}{|l|r|}
\hline Bags & 74.1 \\
\hline Dry & 1502.8 \\
\hline Sub + Bags & 874.7 \\
\hline Gmm & 2.423 \\
\hline \multicolumn{2}{|l|}{} \\
\hline Bags & 74.3 \\
\hline Dry & 1531.7 \\
\hline Sub + Bags & 892.1 \\
\hline Gmm & 2.425 \\
\hline
\end{tabular}

\begin{tabular}{|l|r|r|}
\hline Dry & \multicolumn{2}{|c|}{1512.9} \\
\hline Submerged & \multicolumn{2}{|c|}{2144.3} \\
\hline Drying Rack & \multicolumn{2}{|c|}{7334.5} \\
\hline DB 1 & 8859.7 & 1525.2 \\
\hline DB 2 & 8857.4 & 1522.9 \\
\hline DB 3 & 8856.5 & 1522 \\
\hline DB 4 & 8855.7 & 1521.2 \\
\hline DB 5 & 8855.4 & 1520.9 \\
\hline Gmm & \multicolumn{2}{|c|}{2.395} \\
\hline
\end{tabular}

Rice

Dry Back

\section{CoreLok}

Averages $\quad 2.427$

Averages

2.393

Stddev

0.0007

Stddev

0.0028

Averages $\quad 2.424$

Stddev $\quad 0.0012$ 
Table 28 - LZ DATA

LZ

43 LS - 12 Slag - 45 Sand 00 min Curing Time

\begin{tabular}{l}
\multicolumn{2}{c}{ Rice } \\
\begin{tabular}{|l|r|}
\hline Dry & 1510.3 \\
\hline Submerged & 2144 \\
\hline Gmm & 2.431 \\
\hline \multicolumn{2}{|l|}{} \\
\hline Dry & 1517.7 \\
\hline Submerged & 2149.1 \\
\hline Gmm & 2.434 \\
\hline
\end{tabular}
\end{tabular}

\begin{tabular}{|c|c|c|}
\hline \multicolumn{3}{|c|}{ Dry Back } \\
\hline Dry & \multicolumn{2}{|c|}{1510.3} \\
\hline Submerged & \multicolumn{2}{|c|}{2144} \\
\hline Drying Rack & \multicolumn{2}{|c|}{7334.9} \\
\hline DB 1 & 8850.3 & 1515.4 \\
\hline DB 2 & 8847.7 & 1512.8 \\
\hline DB 3 & 8846.6 & 1511.7 \\
\hline DB 4 & 8846.2 & 1511.3 \\
\hline DB 5 & & \\
\hline $\mathrm{Gmm}$ & \multicolumn{2}{|c|}{2.427} \\
\hline
\end{tabular}

CoreLok
\begin{tabular}{|l|r|}
\hline Bags & 73.8 \\
\hline Dry & 1440 \\
\hline Sub + Bags & 839.6 \\
\hline Gmm & 2.430 \\
\hline \multicolumn{2}{|l|}{} \\
\hline Bags & 73.6 \\
\hline Dry & 1588.9 \\
\hline Sub + Bags & 927.1 \\
\hline Gmm & 2.430 \\
\hline
\end{tabular}

\begin{tabular}{|l|c|c|}
\hline Dry & \multicolumn{2}{|c|}{1517.7} \\
\hline Submerged & \multicolumn{2}{|c|}{2149.1} \\
\hline Drying Rack & \multicolumn{2}{|c|}{6312.5} \\
\hline DB 1 & 7835.5 & 1523 \\
\hline DB 2 & 7834.4 & 1521.9 \\
\hline DB 3 & 7833.7 & 1521.2 \\
\hline DB 4 & 7833.3 & 1520.8 \\
\hline DB 5 & \multicolumn{3}{|c|}{} \\
\hline Gmm & \multicolumn{2}{|c|}{2.422} \\
\hline
\end{tabular}

Rice

Dry Back

CoreLok

Averages $\quad 2.432$

Averages

2.424

Stddev

0.0037

Averages $\quad 2.430$

Stddev $\quad 0.0004$ 
Table 29 - MZ DATA

MZ

28 LS - 27 Slag - 45 Sand 00 min Curing Time

\begin{tabular}{|l|r|}
\multicolumn{2}{c}{ Rice } \\
\hline Dry & 1510 \\
\hline Submerged & 2142.8 \\
\hline Gmm & 2.427 \\
\hline \multicolumn{2}{|l}{} \\
\hline Dry & 1510.9 \\
\hline Submerged & 2143.1 \\
\hline Gmm & 2.426 \\
\hline
\end{tabular}

\begin{tabular}{|c|c|c|}
\hline \multicolumn{3}{|c|}{ Dry Back } \\
\hline Dry & \multicolumn{2}{|c|}{1510} \\
\hline Submerged & \multicolumn{2}{|c|}{2142.8} \\
\hline Drying Rack & \multicolumn{2}{|c|}{7334.6} \\
\hline DB 1 & 8854.5 & 1519.9 \\
\hline DB 2 & 8853 & 1518.4 \\
\hline DB 3 & 8852 & 1517.4 \\
\hline DB 4 & 8851.4 & 1516.8 \\
\hline DB 5 & & \\
\hline $\mathrm{Gmm}$ & \multicolumn{2}{|c|}{2.401} \\
\hline
\end{tabular}

\begin{tabular}{|l|r|}
\hline Bags & 74.3 \\
\hline Dry & 1512.1 \\
\hline Sub + Bags & 880.1 \\
\hline Gmm & 2.423 \\
\hline \multicolumn{2}{|l|}{} \\
\hline Bags & 73.7 \\
\hline Dry & 1512 \\
\hline Sub + Bags & 881 \\
\hline Gmm & 2.427 \\
\hline
\end{tabular}

\begin{tabular}{|l|r|r|}
\hline Dry & \multicolumn{2}{|c|}{1510.9} \\
\hline Submerged & \multicolumn{2}{|c|}{2143.1} \\
\hline Drying Rack & \multicolumn{2}{|c|}{6312.2} \\
\hline DB 1 & 7831.6 & 1519.4 \\
\hline DB 2 & 7830.2 & 1518 \\
\hline DB 3 & 7829.5 & 1517.3 \\
\hline DB 4 & & \\
\hline DB 5 & \multicolumn{3}{|c|}{} \\
\hline Gmm & \multicolumn{2}{|c|}{2.401} \\
\hline
\end{tabular}

Rice

Dry Back

\section{CoreLok}

Averages $\quad 2.426$

Averages

2.401

Stddev

0.0006

Stddev

0.0005

Averages $\quad 2.425$

Stddev $\quad 0.0025$ 
Table 30 - HZ DATA

$\mathrm{HZ}$

13 LS - 42 Slag - 45 Sand 00 min Curing Time

\begin{tabular}{l}
\multicolumn{2}{c}{ Rice } \\
\begin{tabular}{|l|r|}
\hline Dry & 1512.8 \\
\hline Submerged & 2137 \\
\hline Gmm & 2.398 \\
\hline Dry & 1505.5 \\
\hline Submerged & 2134 \\
\hline Gmm & 2.403 \\
\hline
\end{tabular}
\end{tabular}

\begin{tabular}{|l|c|c|}
\multicolumn{2}{c|}{ Dry Back } \\
\hline Dry & \multicolumn{2}{|c|}{1512.8} \\
\hline Submerged & \multicolumn{2}{|c|}{2137} \\
\hline Drying Rack & \multicolumn{2}{|c|}{7334.4} \\
\hline DB 1 & 8857.4 & 1523 \\
\hline DB 2 & 8853.2 & 1518.8 \\
\hline DB 3 & 8852.2 & 1517.8 \\
\hline DB 4 & 8851.7 & 1517.3 \\
\hline DB 5 & & \\
\hline Gmm & \multicolumn{2}{|c|}{2.381} \\
\hline
\end{tabular}

\begin{tabular}{|c|c|}
\hline \multicolumn{2}{|c|}{ CoreLok } \\
\hline Bags & 74.8 \\
\hline Dry & 1513.5 \\
\hline Sub + Bags & 876.7 \\
\hline $\mathrm{Gmm}$ & 2.407 \\
\hline Bags & 73.8 \\
\hline Dry & 1508.8 \\
\hline Sub + Bags & 871.6 \\
\hline $\mathrm{Gmm}$ & 2.398 \\
\hline
\end{tabular}

\begin{tabular}{|l|r|l|}
\hline Dry & \multicolumn{2}{|c|}{1505.5} \\
\hline Submerged & \multicolumn{2}{|c|}{2134} \\
\hline Drying Rack & \multicolumn{2}{|c|}{6312.2} \\
\hline DB 1 & 7832.8 & 1520.6 \\
\hline DB 2 & 7824 & 1511.8 \\
\hline DB 3 & 7822.1 & 1509.9 \\
\hline DB 4 & 7821.5 & 1509.3 \\
\hline DB 5 & \multicolumn{3}{|c|}{} \\
\hline Gmm & \multicolumn{2}{|c|}{2.389} \\
\hline
\end{tabular}

Rice

Dry Back

CoreLok

Averages $\quad 2.401$

Averages

2.385

Stddev $\quad 0.0034$

Stddev

0.0052

Averages $\quad 2.402$

Stddev $\quad 0.0067$ 
Table 31 - LZM DATA

LZM

43 LS - 12 Slag - 45 Sand 00 min Curing Time $+2 \% M C$

\begin{tabular}{|c|c|}
\hline \multicolumn{2}{|c|}{ Rice } \\
\hline Dry & 1505.4 \\
\hline Submerged & 2144.6 \\
\hline $\mathrm{Gmm}$ & 2.445 \\
\hline Dry & 1517.4 \\
\hline Submerged & 2152.2 \\
\hline $\mathrm{Gmm}$ & 2.447 \\
\hline
\end{tabular}

\begin{tabular}{|l|c|c|}
\multicolumn{2}{c|}{ Dry Back } \\
\hline Dry & \multicolumn{2}{|c|}{1505.4} \\
\hline Submerged & \multicolumn{2}{|c|}{2144.6} \\
\hline Drying Rack & \multicolumn{2}{|c|}{7334.4} \\
\hline DB 1 & 8846.2 & 1511.8 \\
\hline DB 2 & 8845.6 & 1511.2 \\
\hline DB 3 & & \\
\hline DB 4 & & \\
\hline DB 5 & \multicolumn{2}{|c|}{} \\
\hline Gmm & \multicolumn{2}{|c|}{2.422} \\
\hline
\end{tabular}

\begin{tabular}{|l|r|}
\hline Bags & 73.9 \\
\hline Dry & 1520.6 \\
\hline Sub + Bags & 890.7 \\
\hline Gmm & 2.445 \\
\hline \multicolumn{2}{|l}{} \\
\hline Bags & 73.7 \\
\hline Dry & 1505.8 \\
\hline Sub + Bags & 882.6 \\
\hline Gmm & 2.447 \\
\hline
\end{tabular}

\begin{tabular}{|l|c|c|}
\hline Dry & \multicolumn{2}{|c|}{1517.4} \\
\hline Submerged & \multicolumn{2}{|c|}{2152.2} \\
\hline Drying Rack & \multicolumn{2}{|c|}{6312.2} \\
\hline DB 1 & 7845.6 & 1533.4 \\
\hline DB 2 & 7838.1 & 1525.9 \\
\hline DB 3 & 7836.5 & 1524.3 \\
\hline DB 4 & 7835.9 & 1523.7 \\
\hline DB 5 & \multicolumn{3}{|c|}{} \\
\hline Gmm & \multicolumn{2}{|c|}{2.422} \\
\hline
\end{tabular}

Rice

Dry Back

CoreLok

Averages $\quad 2.446$

Averages

2.422

Stddev

0.0002

Averages $\quad 2.446$

Stddev $\quad 0.0018$ 
Table 32 - MZM DATA

MZM

28 LS - 27 Slag - 45 Sand + 2\%MC 00min curing

\begin{tabular}{|c|c|}
\hline \multicolumn{2}{|c|}{ Rice } \\
\hline Dry & 1503 \\
\hline Submerged & 2142.4 \\
\hline $\mathrm{Gmm}$ & 2.442 \\
\hline Dry & 1519 \\
\hline Submerged & 2150.8 \\
\hline $\mathrm{Gmm}$ & 2.437 \\
\hline
\end{tabular}

\begin{tabular}{|c|c|c|}
\hline \multicolumn{3}{|c|}{ Dry Back } \\
\hline Dry & \multicolumn{2}{|c|}{1503} \\
\hline Submerged & \multicolumn{2}{|c|}{2142.4} \\
\hline Drying Rack & \multicolumn{2}{|c|}{7334.5} \\
\hline DB 1 & 8851.7 & 1517.2 \\
\hline DB 2 & 8849.4 & 1514.9 \\
\hline DB 3 & 8848.8 & 1514.3 \\
\hline DB 4 & 8848.2 & 1513.7 \\
\hline DB 5 & & \\
\hline $\mathrm{Gmm}$ & \multicolumn{2}{|c|}{2.400} \\
\hline
\end{tabular}

CoreLok
\begin{tabular}{|l|r|}
\hline Bags & 74.5 \\
\hline Dry & 1505 \\
\hline Sub + Bags & 878.1 \\
\hline Gmm & 2.432 \\
\hline Bags & 74.8 \\
\hline Dry & 1518.8 \\
\hline Sub + Bags & 888.3 \\
\hline Gmm & 2.440 \\
\hline
\end{tabular}

\begin{tabular}{|l|c|l|}
\hline Dry & \multicolumn{2}{|c|}{1519} \\
\hline Submerged & \multicolumn{2}{|c|}{2150.8} \\
\hline Drying Rack & \multicolumn{2}{|c|}{6313.3} \\
\hline DB 1 & 7849.4 & 1536.1 \\
\hline DB 2 & 7844.5 & 1531.2 \\
\hline DB 3 & 7842.9 & 1529.6 \\
\hline DB 4 & 7841.4 & 1528.1 \\
\hline DB 5 & 7840.8 & 1527.5 \\
\hline Gmm & \multicolumn{2}{|c|}{2.405} \\
\hline
\end{tabular}

Rice

Dry Back

\section{Corelok}

Averages $\quad 2.439$

Averages

2.402

Stddev

0.0029

Stddev

0.0034

Averages $\quad 2.436$

Stddev $\quad 0.0058$ 
Table 33 - HZM DATA

HZM

13 LS - 42 Slag - 45 Sand + 2\%MC 00min curing

\begin{tabular}{|l|r|}
\multicolumn{2}{c}{ Rice } \\
\hline Dry & 1508.1 \\
\hline Submerged & 2144 \\
\hline Gmm & 2.436 \\
\hline \multicolumn{2}{|l}{} \\
\hline Dry & 1516.8 \\
\hline Submerged & 2147.5 \\
\hline Gmm & 2.430 \\
\hline
\end{tabular}

\begin{tabular}{|l|r|r|}
\multicolumn{3}{c}{ Dry Back } \\
\hline Dry & \multicolumn{2}{|c|}{1508.1} \\
\hline Submerged & \multicolumn{2}{|c|}{2144} \\
\hline Drying Rack & \multicolumn{2}{|c|}{6313.2} \\
\hline DB 1 & 7843.8 & 1530.6 \\
\hline DB 2 & 7841.4 & 1528.2 \\
\hline DB 3 & 7840 & 1526.8 \\
\hline DB 4 & 7838.2 & 1525 \\
\hline DB 5 & 7837.6 & 1524.4 \\
\hline Gmm & \multicolumn{2}{|c|}{2.373} \\
\hline
\end{tabular}

\begin{tabular}{|c|c|}
\hline \multicolumn{2}{|c|}{ CoreLok } \\
\hline Bags & 74.4 \\
\hline Dry & 1510 \\
\hline Sub + Bags & 880.3 \\
\hline $\mathrm{Gmm}$ & 2.429 \\
\hline Bags & 74.4 \\
\hline Dry & 1516 \\
\hline Sub + Bags & 885.3 \\
\hline $\mathrm{Gmm}$ & 2.434 \\
\hline
\end{tabular}

\begin{tabular}{|l|r|r|}
\hline Dry & \multicolumn{2}{|c|}{1516.8} \\
\hline Submerged & \multicolumn{2}{|c|}{2147.5} \\
\hline Drying Rack & \multicolumn{2}{|c|}{7334.6} \\
\hline DB 1 & 8872.5 & 1537.9 \\
\hline DB 2 & 8870.6 & 1536 \\
\hline DB 3 & 8869.3 & 1534.7 \\
\hline DB 4 & 8868.2 & 1533.6 \\
\hline DB 5 & 8867.8 & 1533.2 \\
\hline Gmm & \multicolumn{2}{|c|}{2.367} \\
\hline
\end{tabular}

Rice

Dry Back

CoreLok

Averages

2.433

Averages

2.370

Stddev

0.0045

Stddev

0.0043

Averages 2.431

Stddev $\quad 0.0034$ 
Table 34 - LP DATA

$$
\begin{gathered}
\text { LP } \\
43 \text { LS - } 12 \text { Slag - } 45 \text { Sand }+0.5 \% \text { AC }
\end{gathered}
$$

\begin{tabular}{|l|r|}
\hline Dry & 1511.4 \\
\hline Submerged & 2143.6 \\
\hline Gmm & 2.427 \\
\hline \multicolumn{2}{|l|}{} \\
\hline Dry & 1512.3 \\
\hline Submerged & 2146.2 \\
\hline Gmm & 2.435 \\
\hline
\end{tabular}

\begin{tabular}{|l|c|c|}
\multicolumn{2}{|c|}{ Dry Back } \\
\hline Dry & \multicolumn{2}{|c|}{1511.4} \\
\hline Submerged & \multicolumn{2}{|c|}{2143.6} \\
\hline Drying Rack & \multicolumn{2}{|c|}{6312.6} \\
\hline DB 1 & 7835.8 & 1523.2 \\
\hline DB 2 & 7828.9 & 1516.3 \\
\hline DB 3 & 7826.3 & 1513.7 \\
\hline DB 4 & 7825.3 & 1512.7 \\
\hline DB 5 & 7824.8 & 1512.2 \\
\hline Gmm & \multicolumn{2}{|c|}{2.424} \\
\hline
\end{tabular}

\begin{tabular}{|l|r|}
\hline Bags & 73.8 \\
\hline Dry & 1506.7 \\
\hline Sub + Bags & 880 \\
\hline Gmm & 2.435 \\
\hline \multicolumn{2}{|l|}{} \\
\hline Bags & 73.7 \\
\hline Dry & 1514.4 \\
\hline Sub + Bags & 882.3 \\
\hline Gmm & 2.426 \\
\hline
\end{tabular}

\begin{tabular}{|l|c|l|}
\hline Dry & \multicolumn{2}{|c|}{1512.3} \\
\hline Submerged & \multicolumn{2}{|c|}{2146.2} \\
\hline Drying Rack & \multicolumn{2}{|c|}{7334.6} \\
\hline DB 1 & 8853.2 & 1518.6 \\
\hline DB 2 & 8851.5 & 1516.9 \\
\hline DB 3 & 8850.2 & 1515.6 \\
\hline DB 4 & 8849.5 & 1514.9 \\
\hline DB 5 & 8849.2 & 1514.6 \\
\hline Gmm & \multicolumn{3}{|c|}{2.426} \\
\hline
\end{tabular}

Rice

Dry Back

CoreLok

Averages $\quad 2.431$

Averages

2.425

Stddev

0.0016

Averages $\quad 2.431$

Stddev $\quad 0.0062$ 
Table 35 - MP DATA

MP
$9.5 \mathrm{~mm} 28 \mathrm{LS}-27$ Slag - 45 Sand $\quad+0.5 \%$ AC

Rice

\begin{tabular}{l|r|}
\hline Dry & 1514.6 \\
\hline Submerged & 2143.4 \\
\hline Gmm & 2.419 \\
\hline \multicolumn{2}{|l|}{} \\
\hline Dry & 1509.9 \\
\hline Submerged & 2140.2 \\
\hline Gmm & 2.417 \\
\hline
\end{tabular}

Rice

Averages

Stddev

\begin{tabular}{|l|r|r|}
\hline \multicolumn{2}{|c|}{ Dry Back } \\
\hline Dry & \multicolumn{2}{|c|}{1514.6} \\
\hline Submerged & \multicolumn{2}{|c|}{2143.4} \\
\hline Drying Rack & \multicolumn{2}{|c|}{6312.2} \\
\hline DB 1 & 7831.1 & 1518.9 \\
\hline DB 2 & 7829.2 & 1517 \\
\hline DB 3 & 7828.7 & 1516.5 \\
\hline DB 4 & & \\
\hline DB 5 & \multicolumn{2}{|c|}{} \\
\hline Gmm & \multicolumn{2}{|c|}{2.411} \\
\hline
\end{tabular}

\begin{tabular}{|l|r|r|}
\hline Dry & \multicolumn{2}{|c|}{1509.9} \\
\hline Submerged & \multicolumn{2}{|c|}{2140.2} \\
\hline Drying Rack & \multicolumn{2}{|c|}{7334.5} \\
\hline DB 1 & 8859.3 & 1524.8 \\
\hline DB 2 & 8850.2 & 1515.7 \\
\hline DB 3 & 8847.5 & 1513 \\
\hline DB 4 & 8846.2 & 1511.7 \\
\hline DB 5 & 8845.8 & 1511.3 \\
\hline Gmm & \multicolumn{2}{|c|}{2.412} \\
\hline
\end{tabular}

Dry Back

Averages

Stddev

CoreLok
\begin{tabular}{|l|r|}
\hline Bags & 74 \\
\hline Dry & 1525.8 \\
\hline Sub + Bags & 887.1 \\
\hline Gmm & 2.419 \\
\hline \multicolumn{2}{|l}{} \\
\hline Bags & 74.7 \\
\hline Dry & 1499.1 \\
\hline Sub + Bags & 870 \\
\hline Gmm & 2.414 \\
\hline
\end{tabular}

CoreLok

Averages $\quad 2.416$

Stddev $\quad 0.0038$ 
Table 36 - HP DATA

HP
13 LS - 42 Slag - 45 Sand $+0.5 \%$ AC

Rice

\begin{tabular}{|l|r|}
\hline Dry & 1511.4 \\
\hline Submerged & 2135.9 \\
\hline Gmm & 2.397 \\
\hline \multicolumn{2}{|l}{} \\
\hline Dry & 1511.7 \\
\hline Submerged & 2137.5 \\
\hline Gmm & 2.403 \\
\hline
\end{tabular}

Dry Back

\begin{tabular}{|l|r|r|}
\hline \multicolumn{2}{|c|}{ Dry Back } \\
\hline Dry & \multicolumn{2}{|c|}{1511.4} \\
\hline Submerged & \multicolumn{2}{|c|}{2135.9} \\
\hline Drying Rack & \multicolumn{2}{|c|}{7334.5} \\
\hline DB 1 & 8850 & 1515.5 \\
\hline DB 2 & 8848.1 & 1513.6 \\
\hline DB 3 & 8847.5 & 1513 \\
\hline DB 4 & 8847.2 & 1512.7 \\
\hline DB 5 & & \\
\hline Gmm & \multicolumn{2}{|c|}{2.392} \\
\hline
\end{tabular}

CoreLok

\begin{tabular}{|l|r|}
\hline Bags & 74.5 \\
\hline Dry & 1510.4 \\
\hline Sub + Bags & 872.6 \\
\hline Gmm & 2.398 \\
\hline \multicolumn{2}{|l}{} \\
\hline Bags & 74.4 \\
\hline Dry & 1511.9 \\
\hline Sub + Bags & 874.4 \\
\hline Gmm & 2.402 \\
\hline
\end{tabular}

\begin{tabular}{|l|c|c|}
\hline Dry & \multicolumn{2}{|c|}{1511.7} \\
\hline Submerged & \multicolumn{2}{|c|}{2137.5} \\
\hline Drying Rack & \multicolumn{2}{|c|}{6312.2} \\
\hline DB 1 & 7827.4 & 1515.2 \\
\hline DB 2 & 7825.6 & 1513.4 \\
\hline DB 3 & 7825.1 & 1512.9 \\
\hline DB 4 & & \\
\hline DB 5 & \multicolumn{3}{|c|}{} \\
\hline Gmm & \multicolumn{2}{|c|}{2.398} \\
\hline
\end{tabular}

Rice

Dry Back

CoreLok

Averages $\quad 2.400$

Averages

2.395

Stddev

0.0041

Averages $\quad 2.400$

Stddev $\quad 0.0025$ 
Table 37 - LPM DATA

LPM
43 LS - 12 Slag - 45 Sand $+0.5 \% A C+2 \% M C$

\begin{tabular}{|c|c|}
\hline \multicolumn{2}{|c|}{ Rice } \\
\hline Dry & 1509.2 \\
\hline Submerged & 2143.4 \\
\hline $\mathrm{Gmm}$ & 2.431 \\
\hline Dry & 1511.7 \\
\hline Submerged & 2145.5 \\
\hline $\mathrm{Gmm}$ & 2.434 \\
\hline
\end{tabular}

\begin{tabular}{|c|c|c|}
\hline \multicolumn{3}{|c|}{ Dry Back } \\
\hline Dry & \multicolumn{2}{|c|}{1509.2} \\
\hline Submerged & \multicolumn{2}{|c|}{2143.4} \\
\hline Drying Rack & \multicolumn{2}{|c|}{6312.2} \\
\hline DB 1 & 7831.1 & 1518.9 \\
\hline DB 2 & 7827.7 & 1515.5 \\
\hline DB 3 & 7825.8 & 1513.6 \\
\hline DB 4 & 7824.6 & 1512.4 \\
\hline DB 5 & 7824.2 & 1512 \\
\hline $\mathrm{Gmm}$ & \multicolumn{2}{|c|}{2.420} \\
\hline
\end{tabular}

\begin{tabular}{|l|r|}
\hline Bags & 74.3 \\
\hline Dry & 1510.2 \\
\hline Sub + Bags & 879.7 \\
\hline Gmm & 2.426 \\
\hline \multicolumn{2}{|l|}{} \\
\hline Bags & 73.8 \\
\hline Dry & 1509.9 \\
\hline Sub + Bags & 881.2 \\
\hline Gmm & 2.432 \\
\hline
\end{tabular}

\begin{tabular}{|l|c|c|}
\hline Dry & \multicolumn{2}{|c|}{1511.7} \\
\hline Submerged & 2145.56 \\
\hline Drying Rack & \multicolumn{2}{|c|}{7334.5} \\
\hline DB 1 & 8852.5 & 1518 \\
\hline DB 2 & 8851.3 & 1516.8 \\
\hline DB 3 & 8850.1 & 1515.6 \\
\hline DB 4 & 8849.2 & 1514.7 \\
\hline DB 5 & 8848.7 & 1514.2 \\
\hline Gmm & \multicolumn{2}{|c|}{2.424} \\
\hline
\end{tabular}

Rice

Dry Back

\section{CoreLok}

Averages $\quad 2.432$

Averages

2.422

Stddev

0.0027

Averages $\quad 2.429$

Stddev $\quad 0.0045$ 
Table 38 - MPM DATA

MPM

28 LS - 27 Slag - 45 Sand $+0.5 \%$ AC $+2 \%$ MC

\begin{tabular}{l}
\multicolumn{2}{c}{ Rice } \\
\begin{tabular}{|l|r|}
\hline Dry & 1510.7 \\
\hline Submerged & 2140.2 \\
\hline Gmm & 2.415 \\
\hline \multicolumn{2}{|l}{} \\
\hline Dry & 1509.5 \\
\hline Submerged & 2142.9 \\
\hline Gmm & 2.428 \\
\hline
\end{tabular}
\end{tabular}

\begin{tabular}{|c|c|c|}
\hline \multicolumn{3}{|c|}{ Dry Back } \\
\hline Dry & \multicolumn{2}{|c|}{1510.7} \\
\hline Submerged & \multicolumn{2}{|c|}{2140.2} \\
\hline Drying Rack & \multicolumn{2}{|c|}{7334.4} \\
\hline DB 1 & 8855.1 & 1520.7 \\
\hline DB 2 & 8852.1 & 1517.7 \\
\hline DB 3 & 8850.6 & 1516.2 \\
\hline DB 4 & 8850.1 & 1515.7 \\
\hline DB 5 & & \\
\hline $\mathrm{Gmm}$ & \multicolumn{2}{|c|}{2.396} \\
\hline
\end{tabular}

\begin{tabular}{|l|r|}
\hline Bags & 74.1 \\
\hline Dry & 1514.4 \\
\hline Sub + Bags & 882.1 \\
\hline Gmm & 2.426 \\
\hline \multicolumn{2}{|l|}{} \\
\hline Bags & 73.7 \\
\hline Dry & 1511.8 \\
\hline Sub + Bags & 880.6 \\
\hline Gmm & 2.426 \\
\hline
\end{tabular}

\begin{tabular}{|l|c|c|}
\hline Dry & \multicolumn{2}{|c|}{1509.5} \\
\hline Submerged & \multicolumn{2}{|c|}{2142.9} \\
\hline Drying Rack & \multicolumn{2}{|c|}{6312.2} \\
\hline DB 1 & 7834.5 & 1522.3 \\
\hline DB 2 & 7830.8 & 1518.6 \\
\hline DB 3 & 7828.5 & 1516.3 \\
\hline DB 4 & 7827.5 & 1515.3 \\
\hline DB 5 & 7827.2 & 1515 \\
\hline Gmm & \multicolumn{2}{|c|}{2.407} \\
\hline
\end{tabular}

Rice

Dry Back

\section{CoreLok}

Averages $\quad 2.422$

Averages

2.402

Stddev

0.0078

Averages 2.426

Stddev $\quad 0.0000$ 
Table 39 - HPM DATA

\begin{tabular}{|c|c|c|c|c|c|c|}
\hline \multicolumn{7}{|c|}{ HPM } \\
\hline \multicolumn{7}{|c|}{13 LS - 42 Slag - 45 Sand + .5\% } \\
\hline \multicolumn{2}{|c|}{ Rice } & \multicolumn{3}{|c|}{ Dry Back } & \multicolumn{2}{|c|}{ CoreLok } \\
\hline Dry & 1513.1 & Dry & \multicolumn{2}{|c|}{1513.1} & Bags & 74.7 \\
\hline Submerged & 2137.7 & Submerged & \multicolumn{2}{|c|}{2137.7} & Dry & 1519.3 \\
\hline $\mathrm{Gmm}$ & 2.400 & Drying Rack & \multicolumn{2}{|c|}{7335.4} & Sub + Bags & 880.4 \\
\hline & DB 1 & \multirow{2}{*}{$\begin{array}{l}8861 \\
8858\end{array}$} & 1525.6 & Gmm & 2.408 \\
\hline Dry & 1507 & DB 2 & & 1522.6 & \multirow[b]{2}{*}{ Bags } & \multirow[b]{2}{*}{74.8} \\
\hline Submerged & 2135.6 & DB 3 & 8857.1 & 1521.7 & & \\
\hline $\mathrm{Gmm}$ & 2.406 & DB 4 & 8856.3 & 1520.9 & Dry & 1507.5 \\
\hline & & DB 5 & 8855.9 & 1520.5 & \multirow{2}{*}{\begin{tabular}{|l} 
Sub + Bags \\
Gmm \\
\end{tabular}} & 873.7 \\
\hline & & $\mathrm{Gmm}$ & \multicolumn{2}{|c|}{2.372} & & 2.409 \\
\hline & & Dry & \multicolumn{2}{|c|}{1507} & & \\
\hline & & Submerged & \multicolumn{2}{|c|}{2135.6} & & \\
\hline & & Drying Rack & \multicolumn{2}{|c|}{6312.2} & & \\
\hline & & DB 1 & 7835.9 & 1523.7 & & \\
\hline & & DB 2 & 7830.7 & 1518.5 & & \\
\hline & & DB 3 & 7828.3 & 1516.1 & & \\
\hline & & DB 4 & 7827.5 & 1515.3 & & \\
\hline & & DB 5 & 7827.1 & 1514.9 & & \\
\hline & & $\mathrm{Gmm}$ & \multicolumn{2}{|c|}{2.376} & & \\
\hline \multicolumn{2}{|c|}{ Rice } & \multicolumn{3}{|c|}{ Dry Back } & \multicolumn{2}{|c|}{ CoreLok } \\
\hline Averages & 2.403 & Averages & 2.3 & 74 & Averages & 2.409 \\
\hline Stddev & 0.0040 & Stddev & 0.0 & 25 & Stddev & 0.0006 \\
\hline
\end{tabular}

